\title{
A CONVERGENT ADAPTIVE FINITE ELEMENT METHOD FOR AN OPTIMAL DESIGN PROBLEM*
}

\author{
SÖREN BARTELS AND CARSTEN CARSTENSEN
}

\begin{abstract}
The optimal design problem for maximal torsion stiffness of an infinite bar of given geometry and unknown distribution of two materials of prescribed amounts is one model example in topology optimisation. It eventually leads to a degenerated convex minimisation problem. The numerical analysis is therefore delicate for possibly multiple primal variables $u$ but unique derivatives $\sigma:=D W(D u)$. Even sharp a posteriori error estimates still suffer from the reliability-efficiency gap. However, it motivates a simple edgebased adaptive mesh-refining algorithm (AFEM) that is not a priori guaranteed to refine everywhere. Its convergence proof is therefore based on energy estimates and some refined convexity control. Numerical experiments illustrate even nearly optimal convergence rates of the proposed adaptive finite element method (AFEM).
\end{abstract}

\section{INTRODUCTION}

The optimal design of two materials with given amounts to fill a given domain for a maximal torsion stiffness has attracted much attention since the pioneering analysis of Kohn and Strang, cf. [1, 14] for the setting of topology optimization and $[23,24,20,16,17,10,12]$ for mathematical and numerical studies. The mathematical modelling (outlined in Section 2) leads to generalised solutions characterised by some convexified minimisation problem

$$
\min _{v \in H_{0}^{1}(\Omega)} \int_{\Omega} \psi(|D v|) d x-\int_{\Omega} v d x .
$$

For parameters $0<t_{1}<t_{2}$ and $0<\mu_{1}<\mu_{2}$ with $t_{1} \mu_{2}=t_{2} \mu_{1}$, the energy density function $\psi:[0, \infty) \rightarrow \mathbb{R}$ is defined by $\psi(0)=\lambda \Theta\left(\mu_{1}-\mu_{2}\right)$ for given numbers $\lambda, \Theta \in \mathbb{R}$ and

$$
\psi^{\prime}(t):= \begin{cases}\mu_{2} t & \text { for } 0 \leq t \leq t_{1} \\ t_{1} \mu_{2}=t_{2} \mu_{1} & \text { for } t_{1} \leq t \leq t_{2} \\ \mu_{1} t & \text { for } t_{2} \leq t\end{cases}
$$

The purpose of this paper is to devise an adaptive algorithm and to analyse it in the spirit of $[3,4,15,21,22,25]$. This adaptive finite element method (AFEM) for (1.1) consists of loops of the form

$$
\mathrm{SOLVE} \rightarrow \text { ESTIMATE } \rightarrow \text { MARK } \rightarrow \text { REFINE. }
$$

Because the right-hand side is constant, all data oscillation terms vanish and we are led to refined estimates for the stress error with edge contributions only.

Throughout this paper, the energy density $W: \mathbb{R}^{n} \rightarrow \mathbb{R}$ reads $W(A):=\psi(|A|)$ and its newly established convexity control property (see Proposition 4.2) reads

$$
|D W(A)-D W(B)|^{2} \lesssim W(B)-W(A)-D W(A) \cdot(B-A) \quad \text { for all } A, B \in \mathbb{R}^{n} .
$$

\footnotetext{
Key words and phrases. convergence, adaptive algorithm, optimal design problem.

* Supported by the DFG Research Center MATHEON "Mathematics for key technologies" in Berlin.
} 
It is known that exact and discrete minimizers $u$ and $u_{\ell}$ are (possibly) non-unique, while

$$
\sigma:=D W(D u) \text { and } \sigma_{\ell}:=D W\left(D u_{\ell}\right)
$$

are unique [12]. Theorem 4.3 below refines [12] and shows

$$
\left\|\sigma-\sigma_{\ell}\right\|_{L^{2}(\Omega)} \lesssim\left(\sum_{E \in \mathcal{E}_{\ell}} \eta_{E}^{2}\right)^{1 / 2}
$$

for the edge jumps

$$
\eta_{E}:=h_{E}\left[\sigma_{\ell}\right] \cdot \nu_{E}
$$

of the normal components $\sigma_{\ell} \cdot \nu_{E}$ of the piecewise constant discrete stress $\sigma_{\ell}$ and the size $h_{E}:=|E|$ of the interior edge $E \in \mathcal{E}_{\ell}$ in the triangulation $\mathcal{T}_{\ell}$ of level $\ell$ of the adaptive algorithm.

The main result in Theorem 3.1 in the spirit of $[15,21,22,25]$ (but without data oscillation) guarantees convergence of the adaptive algorithm (AFEM) specified in Section 3. Proofs of that and (1.3)-(1.4) follow in Section 4. Numerical experiments in Section 5 with domains $\Omega$ and meshes $\mathcal{T}_{0}$ from Figure 1.1 conclude the paper. The initial mesh $\mathcal{T}_{0}=\operatorname{red}^{2}\left(\mathcal{T}_{c}\right)$ is obtained from the depicted $\mathcal{T}_{c}$ through two uniform red-refinements. In general, an energy reduction cannot be expected after one refinement of a triangulation.
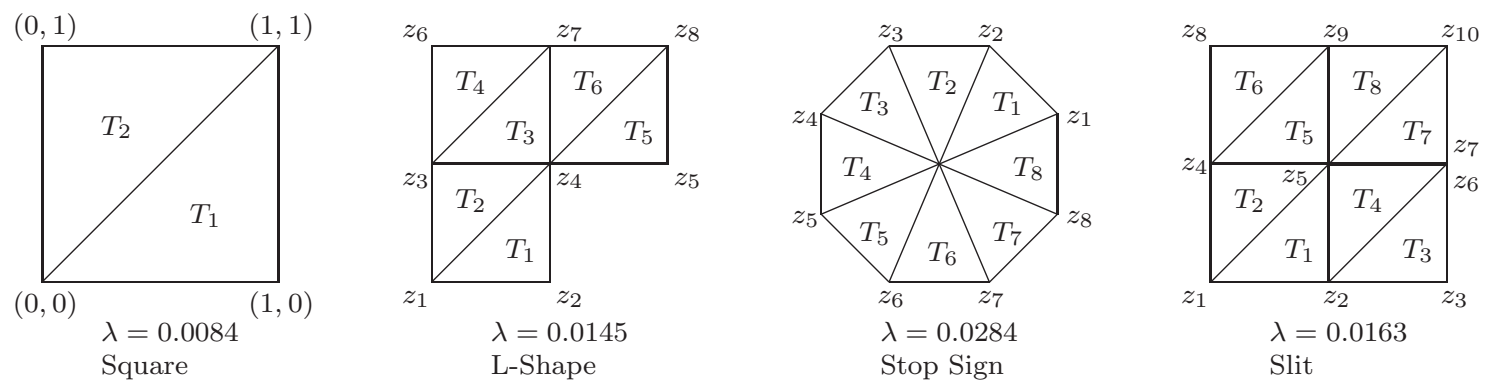

FigURE 1.1. Domains $\Omega_{1}, \ldots, \Omega_{4}$ and corresponding coarse meshes $\mathcal{T}_{c}$ with $\mathcal{T}_{0}:=\operatorname{red}^{2}\left(\mathcal{T}_{c}\right)$ for the numerical experiments with $(\mathrm{AFEM})$.

Example 1.1 (Counter-Example for Error and Energy Reduction). Suppose $\bar{\Omega}=\bigcup \mathcal{T}_{0}=\bigcup \mathcal{T}_{1}$ is a regular polygon, decomposed in the regular triangulations $\mathcal{T}_{0}$ and $\mathcal{T}_{1}=\operatorname{bisec} 3\left(\mathcal{T}_{1}\right)$ as depicted in Figure 1.2. Then $\sigma_{0}=\sigma_{1}$ for the minimizers $u_{0}=u_{1}$ of $(1.1)$ in $\mathcal{P}_{1}\left(\mathcal{T}_{0}\right) \cap H_{0}^{1}(\Omega)$ and $\mathcal{P}_{1}\left(\mathcal{T}_{1}\right) \cap H_{0}^{1}(\Omega)$, respectively.

Proof. To sketch the proof notice that $\left.\sigma_{0}\right|_{T_{j}}$ is parallel to $\left.D \varphi\right|_{T_{j}}$ for the globally continuous and $\mathcal{T}_{0}$-elementwise affine $\varphi \in \mathcal{P}_{1}\left(\mathcal{T}_{0}\right) \cap H_{0}^{1}(\Omega)$ defined by $\varphi(A)=1$ plus $\varphi=0$ along $\partial \Omega$. Since $\sigma_{0}=D W\left(D u_{0}\right)$ there holds

$$
\left.\sigma_{0}\right|_{T_{j}}=\left.s D \varphi\right|_{T_{j}}
$$

for some $s \in \mathbb{R}$ independent of $j=1,2, \ldots, k$. Equilibrium for $u_{0}=s \varphi$ yields

$$
\int_{T_{j}} \sigma_{0} \cdot D \varphi d x-\int_{T_{j}} \varphi d x=0
$$

and $s=h^{2} / 3$ for $h=\left|A-C_{1}\right|=\left|A-C_{2}\right|$ as in Figure 1.3. It remains to prove that

$$
\int_{\Omega} D \varphi_{E} \cdot \sigma_{0} d x=\int_{\Omega} \varphi_{E} d x
$$



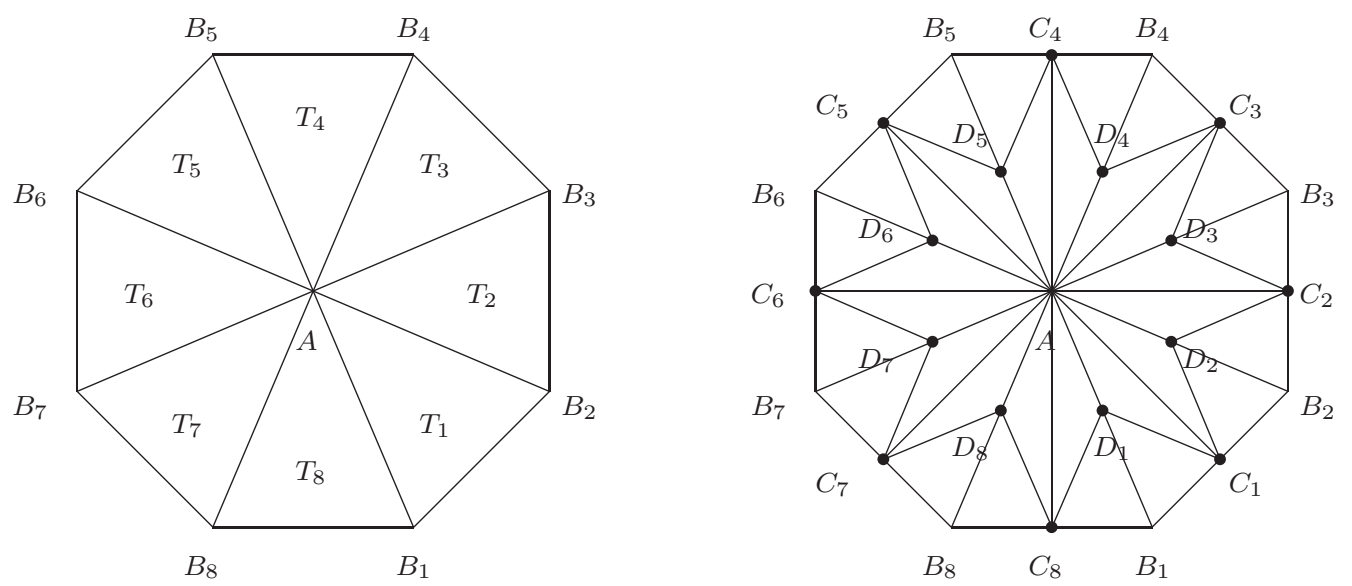

FiguRE 1.2. Triangulation $\mathcal{T}_{0}=\left\{T_{1}, \ldots, T_{k}\right\}$ (left) and $\mathcal{T}_{1}=\operatorname{bisec} 3\left(\mathcal{T}_{0}\right)$ (right) into congruent triangles with vertex $A$ in the center, which is the only free node and the newest vertex in $\mathcal{T}_{0}$.

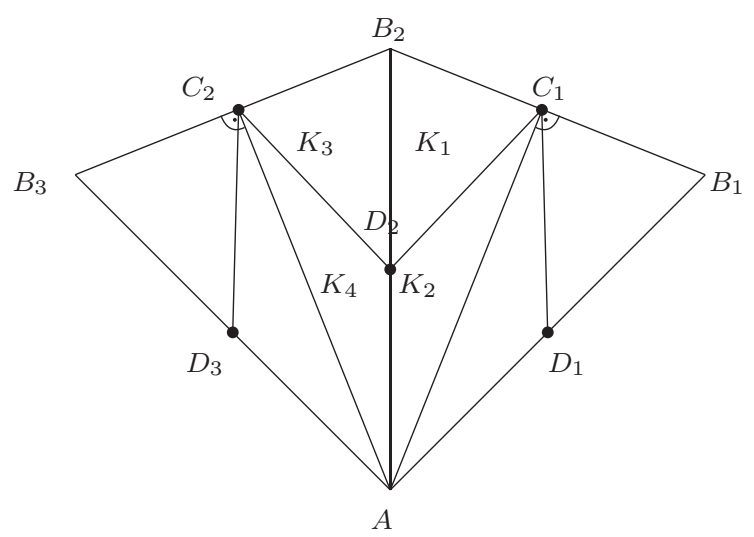

Figure 1.3. Support $\bar{\omega}_{E}=K_{1} \cup \ldots \cup K_{4}$ with $E=\partial T_{1} \cap \partial T_{2}=\operatorname{conv}\left\{A, B_{2}\right\}$ and $\varphi_{E} \in \mathcal{P}_{1}\left(\mathcal{T}_{1}\right) \cap H_{0}^{1}\left(\omega_{E}\right)$ defined by $\varphi_{E}\left(D_{2}\right)=1$ and $\varphi_{E}=0$ on $\partial \omega_{E}$.

for any interior edge $E$ in $\mathcal{T}_{0}$ and corresponding nodal basis function $\varphi_{E} \in \mathcal{P}_{1}\left(\mathcal{T}_{1}\right) \cap H_{0}^{1}(\Omega)$. Adopt notation from Figure 1.3 for $E=\operatorname{conv}\left\{A, B_{2}\right\}$. Then $\varphi_{E}$ is the nodal basis function of $D_{2}$ in $\mathcal{T}_{1}$ and $\left.D \varphi_{E}\right|_{K_{1}}$ is parallel to the straight line through $A$ and $C_{1}$, while $\left.D \varphi_{E}\right|_{K_{2}}$ is parallel to that through $B_{1}$ and $B_{2}$. Since $\left.\sigma_{0}\right|_{K_{1} \cup K_{2}}$ is parallel to the straight line through $A$ and $C_{1}$, it is orthogonal to the edge conv $\left\{B_{1}, B_{2}\right\}$. Consequently, with $\left|D \varphi_{E}\right|_{K_{1}} \mid=2 h^{-1}$,

$$
\begin{aligned}
\int_{T_{1}} \sigma_{0} \cdot D \varphi_{E} d x & =\int_{T_{1}} s D \varphi \cdot D \varphi_{E} d x \\
& =s\left|K_{1}\right| 2 h^{-2}=(2 / 3)\left|K_{1}\right|=\left|T_{1}\right| / 6=\int_{T_{1}} \varphi_{E} d x
\end{aligned}
$$


The same formula holds when $T_{1}$ and $K_{1}$ are replaced by $T_{2}$ and $K_{3}$, respectively. The two formulae prove

$$
\int_{\Omega} \sigma_{0} \cdot D \varphi_{E} d x=\int_{\Omega} \varphi_{E} d x
$$

Since $E$ is arbitrary, it follows that $\sigma_{0}=\sigma_{1}$ and $u_{0}=u_{1}$.

The example shows that energy reduction may fail without an inner node property of the refinement in step MARK of (AFEM) in Section 3.

\section{An Outline of the Mathematical Modelling}

This section recalls the essential steps for the mathematical modelling of an optimal design problem and its connection to the variational problem

$$
(P) \quad \inf _{v \in V} G(\lambda, v)
$$

for $V:=H_{0}^{1}(\Omega)$ and its supremum over the real parameter $\lambda$ and derives the formula for $G(\lambda, v)$.

2.1. Class of Shear Modulus Variables. Given a simply-connected bounded 2D Lipschitz domain $\Omega \subset \mathbb{R}^{2}$ consider the infinite cylinder $\Omega \times \mathbb{R}$ under torsion. The 3D problem is invariant under translation along the third component and so reduces to a $2 \mathrm{D}$ problem in $\Omega$ as discussed below. The optimal design problem is to maximise the torsion stiffness for an optimal composition of the prescribed section $\Omega$ with two materials of reciprocal shear stiffness $0<\mu_{1}<\mu_{2}<\infty$ located at $\Omega_{1}$ and $\Omega_{2}$,

$$
\Omega=\Omega_{1} \cup \Omega_{2} \text { and } \quad \Omega_{1} \cap \Omega_{2}=\emptyset .
$$

The amounts of the materials are prescribed with some parameter $0<\Theta<1$ and exactly fills the domain (so that a partition is possible), i.e., where $|\cdot|$ denotes the area (the 2D Lebesgue measure)

$$
\left|\Omega_{1}\right|=\Theta|\Omega| \text { and } \quad\left|\Omega_{2}\right|=(1-\Theta)|\Omega|
$$

It turns out that the torsion stiffness depends only on the shear modulus $\mu(x)$ which depends on the material point $x \in \Omega$. In fact, one seeks some shear modulus distribution in the set

$$
\mathcal{M}:=\left\{\mu \in L^{\infty}(\Omega): \mu(x) \in\left\{\mu_{1}, \mu_{2}\right\} \text { for almost every } x \in \Omega\right\}
$$

and this defines $\Omega_{j}:=\left\{x \in \Omega: \mu(x)=\mu_{j}\right\}$ up to sets of measure zero. The optimal design problem is therefore recast to seek some shear modulus distribution in the set

$$
\mathcal{M}_{\Theta}:=\left\{\mu \in \mathcal{M}: \int_{\Omega} \mu(x) d x=\mu_{\Theta}|\Omega|\right\} \quad \text { with } \quad \mu_{\Theta}:=\Theta \mu_{1}+(1-\Theta) \mu_{2}
$$

to model the volume constraint. 
2.2. Torsion Stiffness. Given any $\mu \in \mathcal{M}_{\Theta}$, one requires the torsion stiffness $T$ of the 3D beam with a section $\Omega$ and the non-homogeneous shear modulus $\mu(x)^{-1}$. The reciprocal torsion $T^{-1}$ is also given by a minimisation problem for the $2 \mathrm{D}$ stress vector $\sigma^{2 D}=\left(\sigma_{1}^{2 D}, \sigma_{2}^{2 D}\right)$ in the section $\Omega$,

$$
T^{-1}=\inf _{\sigma^{2 D} \in \Sigma} E / m^{2}
$$

where $E$ is the elastic energy and $m$ is the resulting $2 \mathrm{D}$ moment. For any given $\sigma^{2 D}$,

$$
E=\frac{1}{2} \int_{\Omega} \mu(x)\left|\sigma^{2 D}(x)\right|^{2} d x \text { and } m=\int_{\Omega}\left(x_{2} \sigma_{1}^{2 D}(x)-x_{1} \sigma_{2}^{2 D}(x)\right) d x
$$

(recall that $\sigma_{j}^{2 D}$ and $x_{j}$ denotes the $j$ th component of $\sigma^{2 D}$ and $x$, respectively). The stress field $\sigma^{2 D}$ is admissible, written $\sigma^{2 D} \in \Sigma$, if it belongs to $L^{2}\left(\Omega ; \mathbb{R}^{2}\right)$ and the distributional divergence $\operatorname{div} \sigma^{2 D}$ satisfies equilibrium (which reads in its strong form)

$$
\operatorname{div} \sigma^{2 D}=0 \text { in } \Omega \text { and } \sigma^{2 D} \cdot \nu=0 \text { along } \partial \Omega
$$

(where $\nu$ denotes the exterior unit normal along the boundary $\partial \Omega$ ). In other words, $\sigma^{2 D}$ is divergence free in the simply-connected domain $\Omega$. Hence there exists some $u \in V$ such that

$$
\sigma^{2 D}=\left(-\partial u / \partial x_{2}, \partial u / \partial x_{1}\right)
$$

and this defines a one-to-one relation between $\sigma^{2 D} \in \Sigma$ and $u \in V$. Moreover, a direct substitution followed by an integration by parts (with $u=0$ along $\partial \Omega$ ) leads to

$$
m=-\int_{\Omega}\left(x_{2} \partial u / \partial x_{2}+x_{1} \partial u / \partial x_{1}\right) d x=2 \int_{\Omega} u d x
$$

In conclusion, the reciprocal torsion stiffness reads

$$
8 T^{-1}=\inf _{v \in V} \frac{\int_{\Omega} \mu|D v|^{2} d x}{\left(\int_{\Omega} v d x\right)^{2}} .
$$

From calculus of variations, any minimiser $u \in V$ satisfies the first-order variation in the sense that

$$
\int_{\Omega} \mu D u \cdot D v d x=\left(8 T^{-1} \int_{\Omega} u d x\right) \int_{\Omega} v d x \quad \text { for all } v \in V .
$$

Apparently, any multiple of $u$ is also a minimiser and hence the scaling of $u$ may be fixed. In the sequel we choose the multiple constant such that

$$
8 T^{-1} \int_{\Omega} u d x=1 .
$$

Therefore, any minimiser $u \in V$ satisfying this constraint is a weak solution of

$$
-\operatorname{div}(\mu D u)=1 \text { in } \Omega
$$

and this unique $u$ attains the minimum in

$$
-\frac{T}{16}=\min _{v \in V}\left(\frac{1}{2} \int_{\Omega} \mu|D v|^{2} d x-\int_{\Omega} v d x\right) .
$$


2.3. Optimal Design. According to the previous subsections, let $F(\mu, v)$ be defined for $\mu \in \mathcal{M}$ and $v \in V$ by

$$
F(\mu, v):=\frac{1}{2} \int_{\Omega} \mu|D v|^{2} d x-\int_{\Omega} v d x
$$

Then, the optimal design problem for the maximal torsion stiffness reads: Find the optimal design $\mu$ in

$$
\text { (M) } \inf _{\mu \in \mathcal{M}} \inf _{\ominus \in V} F(\mu, v) .
$$

An analytic solution is known for the open ball where $\Omega_{2}$ is the centered smaller ball and $\Omega_{1}$ is the complementary ring with radius determined by the volume constraint. Otherwise, there may be no classical solutions with a discrete $\mu$ (where discrete means that $\mu$ assumes only the two values $\left.\mu_{1}, \mu_{2}\right)$ and, in fact, sequences of designs in $\mathcal{M}_{\Theta}$ exist with finer and finer oscillations and smaller and smaller values of $\inf _{v \in V} F(\mu, v)$ such that the weak limit of such sequences in $L^{\infty}(\Omega)$ is no longer in $\mathcal{M}_{\Theta}$.

The rest of this subsection outlines the relaxation procedure by Kohn and Strang in the essential steps and solely on the formal level. The deeper functional analytic justification can be found in [19].

In the first step of the reformulations, one replaces the volume constraint in $\mathcal{M}_{\Theta}$ by some Lagrange parameter ansatz where $(M)$ is equivalent to

$$
\sup _{\lambda \in \mathbb{R}} \inf _{\mu \in \mathcal{M}} \inf _{v \in V}\left(F(\mu, v)-\lambda \int_{\Omega}\left(\mu-\mu_{\Theta}\right) d x\right) .
$$

One then replaces the order of the last two infima in step two.

Given any $\lambda \in \mathbb{R}$ and $v \in V$, step three computes

$$
\inf _{\mu \in \mathcal{M}}\left(F(\mu, v)-\lambda \int_{\Omega}\left(\mu-\mu_{\Theta}\right) d x\right)=\int_{\Omega} \tilde{g}_{\lambda}(|D v|) d x-\int_{\Omega} v d x+\lambda|\Omega| \mu_{\Theta}
$$

in a pointwise minimisation with

$$
\tilde{g}_{\lambda}(t):=\min \left\{\left(\frac{1}{2} t^{2}-\lambda\right) \mu_{1},\left(\frac{1}{2} t^{2}-\lambda\right) \mu_{2}\right\} .
$$

That is, given $|D v(x)|$ and $v(x), \mu(x)$ is chosen as the value of $\mu_{1}$ or $\mu_{2}$ which leads to the smaller value

$$
\left(\frac{1}{2}|D v(x)|^{2}-\lambda\right) \mu_{j}
$$

Thus, if $\frac{1}{2}|D v(x)|^{2}-\lambda<0$ then $\mu(x)=\mu_{2}$ and if $\frac{1}{2}|D v(x)|^{2}-\lambda>0$ then $\mu(x)=\mu_{1}$; in case $\frac{1}{2}|D v(x)|^{2}-\lambda=0$ any choice is possible with a vanishing contribution. This leads to the aforementioned formula with $\tilde{g}_{\lambda}$.

Step four considers the problem for fixed $\lambda \in \mathbb{R}$ and

$$
\tilde{G}(\lambda, v)=\int_{\Omega} \tilde{g}_{\lambda}(|D v|) d x-\int_{\Omega} v d x+\lambda \mu_{\Theta}|\Omega|,
$$

namely the nonconvex minimisation problem (for each given parameter $\lambda$ )

$$
\inf _{v \in V} \tilde{G}(\lambda, v) \text {. }
$$

It is well-established in the modern calculus of variations that, owing to the non-convex $\tilde{g}_{\lambda}$ the infimum may in fact not be attained. Nevertheless, the infimal value can equivalently 
be computed as the minimal value of the convexified problem where $\tilde{g}_{\lambda}$ is replaced by its convex hull $g_{\lambda}:=\tilde{g}_{\lambda}^{* *}$. With $t_{1}:=\sqrt{2 \lambda \mu_{1} / \mu_{2}}$ and $t_{2}:=\left(\mu_{2} / \mu_{1}\right) t_{1}$ there holds

$$
g_{\lambda}(t):= \begin{cases}\mu_{2}\left(t^{2} / 2-\lambda\right) & \text { for } t \leq t_{1}, \\ \sqrt{t_{1} t_{2} \mu_{1} \mu_{2}} t-\lambda\left(\mu_{1}+\mu_{2}\right) & \text { for } t_{1} \leq t \leq t_{2}, \\ \mu_{1}\left(t^{2} / 2-\lambda\right) & \text { for } t_{2} \leq t .\end{cases}
$$

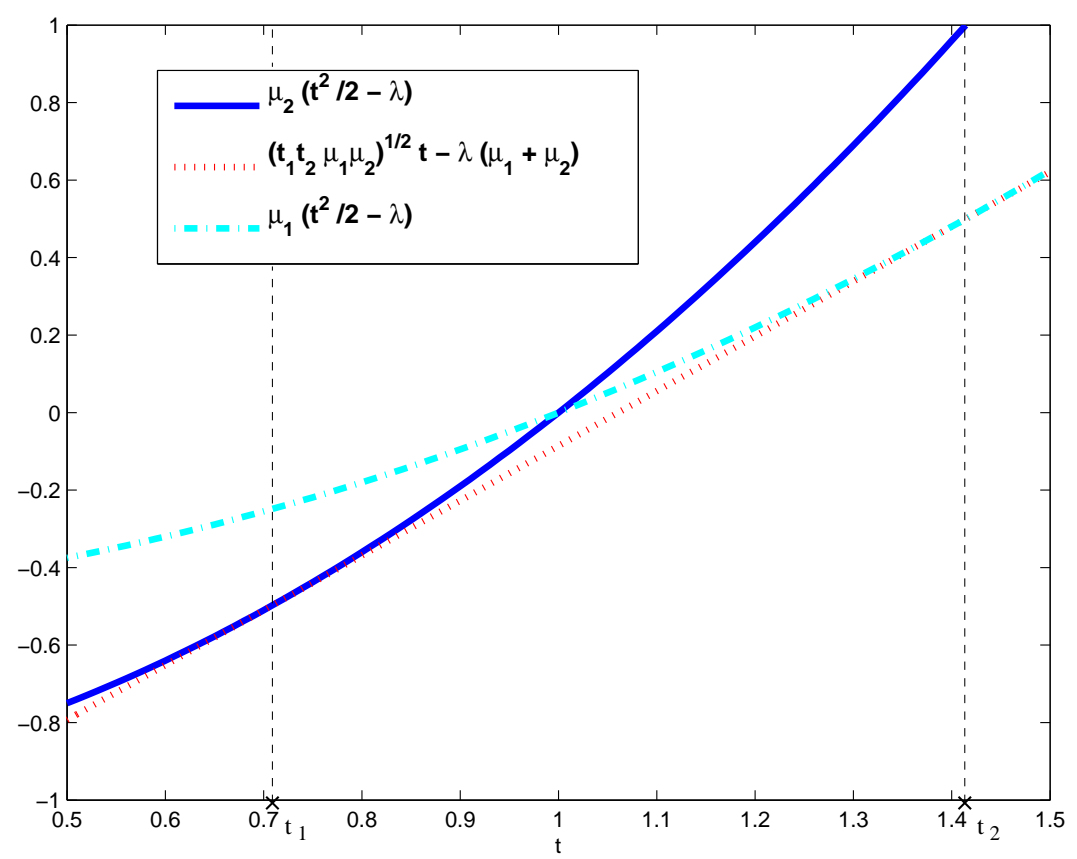

Figure 2.1. Plot of two parabola and one straight line in the definition of $g_{\lambda}(t)$ for $\mu_{1}=1, \mu_{2}=2$, and $\lambda=1 / 2$. For these values it holds that $t_{1}=$ $\sqrt{2 \lambda \mu_{1} / \mu_{2}}=1 / \sqrt{2}$ and $t_{2}=\left(\mu_{2} / \mu_{1}\right) t_{1}=\sqrt{2}$.

Figure 2.1 illustrates the functions $g_{\lambda}$ and $\tilde{g}_{\lambda}$. Altogether, the problem $(M)$ is equivalent to the saddle point problem

$$
\text { (S) } \sup _{\lambda \in \mathbb{R}} \inf _{v \in V} G(\lambda, v) \quad \text { for } G(\lambda, v)=\int_{\Omega} g_{\lambda}(|D v|) d x-\int_{\Omega} v d x+\lambda \mu_{\Theta}|\Omega| .
$$

The main result of Kohn and Strang [19] proves that $(M)$ and $(S)$ are equivalent. The proof therein follows the outline given here and fills the remaining mathematical gaps rigorously. This paper is devoted to the numerical analysis of $(P)$ which results from $(S)$ by freezing the parameter $\lambda$.

\section{AFEM And Its Convergence}

This section states the algorithm (AFEM) to generate self-adapted meshes and discusses its convergence properties.

(AFEM) Input: A coarse regular triangulation $\mathcal{T}_{0}=\operatorname{red}^{2}\left(\mathcal{T}_{c}\right)$ with $\mathcal{T}_{c}$ from Figure 1.1. For $\ell=0,1,2, \ldots$ (until termination) do SOLVE, ESTIMATE, MARK, REFINE to output a sequence 
of discrete spaces $V_{0} \subset V_{1} \subset V_{2} \subset \ldots \subset \cup_{\ell=0}^{\infty} V_{\ell} \subseteq V=H_{0}^{1}(\Omega)$ and discrete minimizers $u_{0}, u_{1}, u_{2}, \ldots$ with associated stress approximations $\sigma_{0}, \sigma_{1}, \sigma_{2}, \ldots$

SOLVE: Given the regular triangulation $\mathcal{T}_{\ell}$ of $\Omega$ into triangles set

$$
\mathcal{P}_{k}\left(\mathcal{T}_{\ell}\right):=\left\{v_{h} \in L^{\infty}(\Omega): \forall T \in \mathcal{T}_{\ell},\left.v_{h}\right|_{T} \text { is a polynomial of total degree } \leq k\right\},
$$

let $\mathcal{N}_{\ell}$ be the union of all vertices of triangles in $\mathcal{T}_{\ell}$ also called nodes, and $\mathcal{E}_{\ell}$ denote the set of all interior edges in $\mathcal{T}_{\ell}$. Compute a discrete minimizer $u_{\ell}$ of $I$ in $V_{\ell}:=\mathcal{P}_{1}\left(\mathcal{T}_{\ell}\right) \cap V$ with Newton-Raphson scheme where $W(\cdot)=\psi(|\cdot|)$ with $\psi$ from (1.2) and

$$
I(v):=\int_{\Omega} W(D v)-\int_{\Omega} v d x \quad \text { for all } v \in V_{\ell}
$$

Set $\sigma_{\ell}:=D W\left(D u_{\ell}\right) \in L^{2}\left(\Omega ; \mathbb{R}^{2}\right)$.

ESTIMATE: Compute $\eta_{E}:=h_{E}^{1 / 2}\left\|\left[\sigma_{\ell}\right] \cdot \nu_{E}\right\|_{L^{2}(E)}$ for all $E \in \mathcal{E}_{\ell}$ and set

$$
\begin{aligned}
\eta_{\mathcal{E}}^{(\ell)} & :=\left(\sum_{E \in \mathcal{E}_{\ell}} \eta_{E}^{2}\right)^{1 / 2}, \\
\eta_{A}^{(\ell)} & :=\left\|\sigma_{\ell}-A_{\ell} \sigma_{\ell}\right\|_{L^{2}(\Omega)}, \\
\eta_{G}^{(\ell)} & :=\left\|D u_{\ell}-A_{\ell} D u_{\ell}\right\|_{L^{2}(\Omega)} .
\end{aligned}
$$

Here, given $p_{\ell} \in \mathcal{P}_{0}\left(\mathcal{T}_{\ell}\right)^{2}$, the function $A_{\ell} p_{\ell} \in \mathcal{S}^{1}\left(\mathcal{T}_{\ell}\right)^{2}=\mathcal{P}_{1}\left(\mathcal{T}_{\ell}\right)^{2} \cap H^{1}\left(\Omega ; \mathbb{R}^{2}\right)$ is defined by

$$
\left(A_{\ell} p_{\ell}\right)(z):=\left|\omega_{z}\right|^{-1} \int_{\omega_{z}} p_{\ell} d y
$$

for $z \in \mathcal{N}_{\ell} \cap \Omega$ and $\bar{\omega}_{z}$, with area $\left|\bar{\omega}_{z}\right|$, is the union of all $T \in \mathcal{T}_{\ell}$ with vertex $z$.

MARK: Sort $\mathcal{E}_{\ell}$ in $\left(E_{1}, \ldots, E_{N}\right)$ with $\eta_{E_{1}} \leq \eta_{E_{2}} \leq \ldots \leq \eta_{E_{N}}$ and choose the minimal $k$ with

$$
\frac{1}{2} \eta_{\mathcal{E}}^{(\ell)} \leq\left(\sum_{j=k}^{N} \eta_{E_{j}}^{2}\right)^{1 / 2} .
$$

Set $\mathcal{M}_{\ell}:=\left\{E_{k}, E_{k+1}, \ldots, E_{N}\right\}$

REFINE: Generate refined triangulation $\mathcal{T}_{\ell+1}$ with subordinated finite element space $V_{\ell+1}:=$ $\mathcal{P}_{1}\left(\mathcal{T}_{\ell+1}\right) \cap V \supset V_{\ell}$ such that every triangle $T$ in $\mathcal{T}_{\ell}$ with some edge $E$ in $\mathcal{M}_{\ell}$ is refined by bisec5 in $\mathcal{T}_{\ell+1}$ and the shape regularity and conformity of $\mathcal{T}_{\ell+1}$ is maintained.

More details on REFINE can be found in [7] for 2D triangulations into triangles with refinements from the list depicted in Figure 3.1.

Theorem 3.1. There exists some constant $0<\kappa \leq 1$ (which depends on $\mathcal{T}_{0}$ and $W$ only) such that $\sigma=D W(D u), \sigma_{\ell}=D W\left(D u_{\ell}\right)$ from the Algorithm (AFEM), and

$$
0 \leq \delta_{\ell}:=\min E\left(V_{\ell}\right)-\min E(V)=E\left(u_{\ell}\right)-E(u)
$$

satisfy

$$
\kappa\left(2 \mu_{2}\right)^{-2}\left\|\sigma-\sigma_{\ell}\right\|_{L^{2}(\Omega)}^{4}+\delta_{\ell+1} \leq\left(1-\kappa \delta_{\ell}\right) \delta_{\ell} \text { for } \ell=0,1,2, \ldots
$$




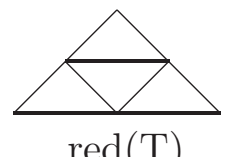

$\operatorname{red}(\mathrm{T})$

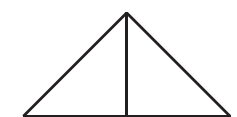

$\operatorname{green}(\mathrm{T})$

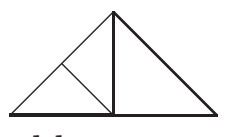

blue $_{\text {left }}$

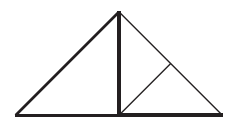

blue $_{\text {right }}$

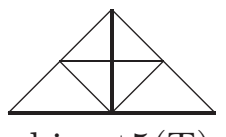

$\operatorname{bisect5}(\mathrm{T})$

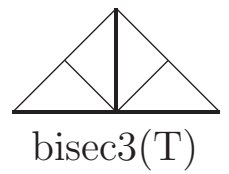

Figure 3.1. Possible refinements of a triangle $T$ in (AFEM).

The proof of Theorem 3.1 follows in Section 4. The interpretation of Theorem 3.1 is that $\left(\delta_{\ell}\right)_{\ell=0,1,2, \ldots}$ behaves like a linearly convergent sequence (with limit zero) as long as $\delta_{\ell} \geq \varepsilon>0$ stays away from zero (with an energy error reduction factor $\rho:=1-\kappa \varepsilon<1$ ). This is called $q$-linear convergence in the preasymptotic range. It follows that

$$
\delta_{\ell} \leq \rho^{\ell} \delta_{0} \text { for all } \ell=0,1,2, \ldots, L
$$

and $L$ such that $\delta_{L}<\varepsilon$. Since $\delta_{\ell} \leq \delta_{\ell+1}$ there holds

$$
\delta_{\ell}<\varepsilon \text { for all } \ell=L, L+1, L+2, \ldots
$$

This and the fact that $\varepsilon>0$ may be chosen arbitrarily small implies convergence

$$
\lim _{\ell \rightarrow \infty} \delta_{\ell}=0
$$

of the energy errors and as a consequence of the stress errors

$$
\lim _{\ell \rightarrow \infty}\left\|\sigma-\sigma_{\ell}\right\|_{L^{2}(\Omega)}=0 .
$$

Moreover, the proof of Theorem 3.1 implies that

$$
\left(2 \mu_{2}\right)^{-2} \sum_{\ell=0}^{\infty}\left\|\sigma-\sigma_{\ell}\right\|_{L^{2}(\Omega)}^{4}+\sum_{\ell=0}^{\infty} \delta_{\ell}^{2} \leq \kappa^{-1} \delta_{0} .
$$

In other words, with the sequence spaces

$$
\ell^{p}:=\left\{\left(x_{j}\right)_{j=0,1,2, \ldots} \in \mathbb{R}^{\mathbb{N}_{0}}: \sum_{j=0}^{\infty}\left|x_{j}\right|^{p}<\infty\right\},
$$

there holds

$$
\left(\left\|\sigma-\sigma_{j}\right\|_{L^{2}(\Omega)}\right)_{j=0,1,2, \ldots} \in \ell^{4} \quad \text { and } \quad\left(\delta_{j}\right)_{j=0,1,2, \ldots} \in \ell^{2}
$$

\section{Proofs}

Theorem 3.1 and the reliability (1.4) is based on the new estimate (1.3) which will be a consequence of the following lemma.

Lemma 4.1. For $\psi$ as in (1.2) and all $a, b \geq 0$ there holds

$$
2(\psi(a)-\psi(b))+b \psi^{\prime}(b)-a \psi^{\prime}(a) \leq \psi^{\prime}(a)^{2}\left(a / \psi^{\prime}(a)-b / \psi^{\prime}(b)\right) .
$$


Proof. Suppose first that $a \geq b$ and let $I:=\left|(a, b) \cap\left(t_{1}, t_{2}\right)\right|$ be the length of the interval $(a, b) \cap\left(t_{1}, t_{2}\right)$. Since $\psi^{\prime}$ is monotone, there holds

$$
(-I)\left(\mu_{2} t_{1}\right)^{2} \leq(-I) \psi^{\prime}(a)^{2} .
$$

Note that (4.1) is trivial if $I=0$ and otherwise follows from $a \leq t_{2}$ and $\psi^{\prime}(a) \leq \psi^{\prime}\left(t_{2}\right)=$ $\mu_{1} t_{2}=\mu_{2} t_{1}$. On defining $\phi(t)=\psi^{\prime}(t) / t$ there holds

$$
t^{2} \phi^{\prime}(t)=\left\{\begin{array}{ll}
0 & \text { for } t \notin\left(t_{1}, t_{2}\right), \\
-\mu_{2} t_{1} & \text { for } t \in\left(t_{1}, t_{2}\right),
\end{array} \quad(1 / \phi(t))^{\prime}= \begin{cases}0 & \text { for } t \notin\left(t_{1}, t_{2}\right), \\
1 /\left(\mu_{2} t_{1}\right) & \text { for } t \in\left(t_{1}, t_{2}\right) .\end{cases}\right.
$$

Therefore, we deduce with (4.1) that

$$
\begin{aligned}
\int_{a}^{b} t^{2} \phi^{\prime}(t) d t & =-I \mu_{2} t_{1} \leq-I \frac{\psi^{\prime}(a)^{2}}{\mu_{2} t_{1}}=-\psi^{\prime}(a)^{2} \int_{a}^{b}(1 / \phi(t))^{\prime} d t \\
& =-\psi^{\prime}(a)^{2}(1 / \phi(b)-1 / \phi(a))=\psi^{\prime}(a)^{2}\left(a / \psi^{\prime}(a)-b / \psi^{\prime}(b)\right) .
\end{aligned}
$$

Integration by parts implies

$$
\begin{aligned}
\int_{a}^{b} t^{2} \phi^{\prime}(t) d t & =-2 \int_{a}^{b} t \phi(t) d t+\left(b^{2} \phi(b)-a^{2} \phi(a)\right)=-2 \int_{a}^{b} \psi^{\prime}(t) d t+\left(b \psi^{\prime}(b)-a \psi^{\prime}(a)\right) \\
& =-2(\psi(b)-\psi(a))+\left(b \psi^{\prime}(b)-a \psi^{\prime}(a)\right) .
\end{aligned}
$$

The combination of the two estimates implies the lemma in case $a \leq b$. If $a>b$ then the choice $I=-\left|(b, a) \cap\left(t_{1}, t_{2}\right)\right|$ and the above argumentation show the lemma.

Proposition 4.2. For $A \in \mathbb{R}^{n}$ let $W(A):=\psi(|A|)$. For any $A, B \in \mathbb{R}^{n}$ there holds

$$
\left(2 \mu_{2}\right)^{-1}|D W(A)-D W(B)|^{2} \leq W(B)-W(A)-D W(A) \cdot(B-A) .
$$

Proof. We abbreviate $a:=|A|$ and $b:=|B|$. Noting that $A \cdot D W(A)=a \psi^{\prime}(a)$ and $B$. $D W(B)=b \psi^{\prime}(b)$ we deduce from Lemma 4.1 that

$$
L H S:=B \cdot D W(B)-A \cdot D W(A)-\psi^{\prime}(a)^{2}\left(a / \psi^{\prime}(a)-b / \psi^{\prime}(b)\right) \leq 2(W(B)-W(A)) .
$$

Using once more that $a \psi^{\prime}(a)=A \cdot D W(A)$ we rewrite the left-hand side as

$$
L H S=2 D W(A) \cdot(B-A)+B \cdot(D W(B)-2 D W(A))+\psi^{\prime}(a)^{2} \frac{b}{\psi^{\prime}(b)} .
$$

Since $B=\left(b / \psi^{\prime}(b)\right) D W(B)$ and $\psi^{\prime}(a)^{2}=|D W(A)|^{2}$ we infer that

$$
B \cdot(D W(B)-2 D W(A))+\psi^{\prime}(a)^{2} \frac{b}{\psi^{\prime}(b)}=\frac{b}{\psi^{\prime}(b)}|D W(A)-D W(B)|^{2} .
$$

A combination of the estimates with $b / \psi^{\prime}(b) \geq 1 / \mu_{2}$ implies the assertion.

Remark 4.1. Proposition 4.2 is sharper than the estimate in $[10,12,13]$, but those are in fact equivalent $[17,18]$.

Theorem 4.3. There hold (1.4) and

$$
\left(2 \mu_{2}\right)^{-1}\left\|\sigma-\sigma_{\ell}\right\|_{L^{2}(\Omega)}^{2}+\delta_{\ell} \lesssim\left(\sum_{E \in \mathcal{E}_{\ell}} \eta_{E}^{2}\right)^{1 / 2}
$$


Proof. For almost all $x \in \Omega$ and $\sigma_{\ell}:=D W(A), A=D u_{\ell}(x)$ and $\sigma:=D W(B), B=D u(x)$, Proposition 4.2 reads

$$
\left(2 \mu_{2}\right)^{-1}\left|\sigma(x)-\sigma_{\ell}(x)\right|^{2} \leq W(D u(x))-W\left(D u_{\ell}(x)\right)-\sigma_{\ell}(x) \cdot D\left(u-u_{\ell}\right)(x) .
$$

The integration over $x \in \Omega$ leads to

$$
\left(2 \mu_{2}\right)^{-1}\left\|\sigma-\sigma_{\ell}\right\|_{L^{2}(\Omega)}^{2}+\delta_{\ell} \leq \mathcal{R} e s_{\ell}\left(u-u_{\ell}\right)
$$

for the residual

$$
\mathcal{R} e s_{\ell}(v):=\int_{\Omega} v d x-\int_{\Omega} \sigma_{\ell} \cdot D v d x
$$

Given this residual, one argues as in the linear situation for the design of explicit residualbased error estimators. With a particular weak Clement-type interpolation operator $J$ from [8] one argues as in $[4,5,9]$ to deduce

$$
\left\|\mathcal{R} e s_{\ell}\right\|_{V^{*}} \lesssim\left(\sum_{E \in \mathcal{E}_{\ell}} \eta_{E}^{2}\right)^{1 / 2}
$$

Note that the right-hand side is constant and hence data oscillation terms vanish. Finally, owing to the reliability-efficiency gap [9], there is no (immediate) control on $\left\|u-u_{\ell}\right\|_{V}$ and hence solely growth conditions are available to guarantee $\|u\|_{V}+\left\|u_{\ell}\right\|_{V} \lesssim 1$. Therefore, the aforementioned estimates and

yield the assertion.

$$
\left\|u-u_{\ell}\right\|_{V} \lesssim 1
$$

Remark 4.2. Theorem 4.3 is sharper than the corresponding results in $[9,12,13]$.

Proof of Theorem 3.1. The residual is the same as for linear elliptic problems, e.g., as for the Poisson problem. Hence the subsequent arguments are well established in [4, 15, 21, 22, 25] and therefore are briefly mentioned. The reliability of the residual-based estimates from Theorem 4.3 and the bulk criterion give

$$
\left(2 \mu_{2}\right)^{-1}\left\|\sigma-\sigma_{\ell}\right\|_{L^{2}(\Omega)}^{2}+\delta_{\ell} \lesssim\left(\sum_{E \in \mathcal{M}_{\ell}} \eta_{E}^{2}\right)^{1 / 2}
$$

where $\eta_{E}:=h_{E}^{1 / 2}\left\|\left[\sigma_{\ell}\right] \cdot \nu_{E}\right\|_{L^{2}(E)}$ is computed with respect to the triangulation $\mathcal{T}_{\ell}$. For each $E$ in $\mathcal{M}_{\ell}$ the step REFINE allows the design a discrete test function $\varphi_{E}$ in $V_{\ell+1}$ with support $\omega_{E}=\operatorname{int}\left(T_{+} \cup T_{-}\right)$for the neighbouring elements $T_{+}, T_{-}$in $\mathcal{T}_{\ell}$ with $E=\partial T_{+} \cap \partial T_{-}$and the properties $\int_{E} \varphi_{E} d s=\frac{1}{2} h_{E}$ and, by the inner node property, $\int_{\omega_{E}} \varphi_{E} d x=0$ plus $\left\|\varphi_{E}\right\|_{V} \approx 1$. Then,with a piecewise integration by parts

$$
\frac{1}{2} \eta_{E}=\int_{E} \varphi_{E}\left[\sigma_{\ell}\right] \cdot \nu_{E} d s=\int_{\omega_{E}} \sigma_{\ell} \cdot D \varphi_{E} d x+\int_{\omega_{E}} \varphi_{E} \operatorname{div}_{h} \sigma_{\ell} d x .
$$

Since the discrete stress is piecewise constant, its piecewise divergence vanishes. Since $\sigma_{\ell+1}$ is in discrete equilibrium with $\varphi_{E} \in V_{\ell+1}$ and right hand side 1 there follows

Consequently,

$$
\int_{\omega_{E}} \sigma_{\ell+1} \cdot D \varphi_{E} d x=\int_{\omega_{E}} \varphi_{E} d x
$$

$$
\frac{1}{2} \eta_{E}=\int_{\omega_{E}}\left(\sigma_{\ell}-\sigma_{\ell+1}\right) \cdot D \varphi_{E} d x .
$$


Since $\left\|\varphi_{E}\right\|_{V} \lesssim 1$ this implies

$$
\eta_{E} \lesssim\left\|\sigma_{\ell+1}-\sigma_{\ell}\right\|_{L^{2}\left(\omega_{E}\right)}
$$

The finite overlap of the edge patches $\left(\omega_{E}: E \in \mathcal{M}_{\ell}\right)$ leads to

$$
\sum_{E \in \mathcal{M}_{\ell}} \eta_{E}^{2} \lesssim\left\|\sigma_{\ell+1}-\sigma_{\ell}\right\|_{L^{2}\left(\omega_{E}\right)}^{2}
$$

On the other hand, $\sigma_{\ell+1}=D W(A), A=D u_{\ell+1}$ and $\sigma_{\ell}=D W(B), B=D u_{\ell}$ lead in Proposition 4.2 to

$$
\left(2 \mu_{2}\right)^{-1}\left\|\sigma_{\ell+1}-\sigma_{\ell}\right\|_{L^{2}(\Omega)}^{2} \leq \int_{\Omega} W\left(D u_{\ell}\right)-W\left(D u_{\ell+1}\right) d x-\int_{\Omega} \sigma_{\ell+1} \cdot D\left(u_{\ell}-u_{\ell+1}\right) d x .
$$

Since $u_{\ell+1}-u_{\ell} \in V_{\ell+1}$ and $\sigma_{\ell+1}$ satisfies the discrete equilibrium condition

$$
\int_{\Omega} \sigma_{\ell+1} \cdot D\left(u_{\ell}-u_{\ell+1}\right) d x=\int_{\Omega}\left(u_{\ell}-u_{\ell+1}\right) d x
$$

the aforementioned estimate reads

$$
\left(2 \mu_{2}\right)^{-1}\left\|\sigma_{\ell+1}-\sigma_{\ell}\right\|_{L^{2}(\Omega)}^{2} \leq \delta_{\ell}-\delta_{\ell+1} .
$$

The combination of (4.3)-(4.5) provides

$$
\begin{aligned}
\left(2 \mu_{2}\right)^{-1}\left\|\sigma-\sigma_{\ell}\right\|_{L^{2}(\Omega)}^{2}+\delta_{\ell} & \lesssim\left(\sum_{E \in \mathcal{M}_{\ell}} \eta_{E}^{2}\right)^{1 / 2} \\
& \lesssim\left\|\sigma_{\ell+1}-\sigma_{\ell}\right\|_{L^{2}(\Omega)} \leq\left(2 \mu_{2}\right)^{1 / 2}\left(\delta_{\ell}-\delta_{\ell+1}\right)^{1 / 2} .
\end{aligned}
$$

In other words, for some constant $C \geq 1$ (which depends on the form of the triangular element domains through the minimal interior angle, on $\mu_{1}$ and on the growth condition $g_{\lambda}$ ), there holds

$$
\left(2 \mu_{2}\right)^{-2}\left\|\sigma-\sigma_{\ell}\right\|_{L^{2}(\Omega)}^{4}+\delta_{\ell}^{2} \leq C\left(\delta_{\ell}-\delta_{\ell+1}\right) .
$$

This is the assertion with $\kappa=C^{-1} \leq 1$.

\section{Numerical Experiments}

This section reports on four numerical experiments defined through the respective domains $\Omega_{1}, \Omega_{2}, \Omega_{3}, \Omega_{4}$ and initial meshes $\mathcal{T}_{0}:=\operatorname{red}^{2}\left(\mathcal{T}_{0}\right)$ plus the eventually computed values of $\lambda$ of Figure 1.1. In all of the examples, $\mu_{1}=1, \mu_{2}=2$, and $\Theta=1 / 2$. The real numbers $t_{1}=\sqrt{2 \lambda \mu_{1} / \mu_{2}}$ and $t_{2}=\left(\mu_{2} / \mu_{1}\right) t_{1}$ are defined through the parameter $\lambda$ (from Figure 1.1) which was determined by a golden section search in the interval $[0,1]$ and a minimization of the energy functional in $V_{\ell}$ with triangulations $\operatorname{red}^{4}\left(\mathcal{T}_{0}\right)$ resulting from four uniform redrefinements (cf. Figure 3.1) of the respective coarse triangulations $\mathcal{T}_{0}$. The sequence of intervals $I_{j}=\left[a_{j}, b_{j}\right]$ provided by the search routine with stopping criterion $b_{j}-a_{j}<10^{-4}$ is for $j=5,6, \ldots, 20$ given (for $j=5,6, \ldots, 12$ in the first and for $j=13,14, \ldots, 20$ in the second row) by:

\begin{tabular}{|c|c|c|c|c|c|c|c|c|}
\hline$a_{j}=$ & 0 & 0 & 0 & 0 & 0 & .005025 & .005025 & .006944 \\
\hline$b_{j}=$ & .090170 & .055728 & .034442 & .021286 & .013156 & .013156 & .010050 & .010050 \\
\hline
\end{tabular}

\begin{tabular}{|c|c|c|c|c|c|c|c|c|}
\hline$a_{j}=$ & .006944 & .007678 & .008131 & .008131 & .008303 & .008411 & .008411 & .008411 \\
\hline$b_{j}=$ & .008864 & .008864 & .008864 & .008584 & .008584 & .008584 & .008518 & .008477 \\
\hline
\end{tabular}


For each new interval end with $j=9,10, \ldots, 20$ (i.e., $\lambda_{j}=0.013156,0.005025,0.010050, \ldots$ ) we plotted the obtained solution of the minimization problem in Figures 5.1 and 5.2. After 20 iterations the numerical solution has approximately the right volume fraction $1 / 2$ for each of the two materials with a transition region separating them.

Each of the domains $\Omega_{1}, \Omega_{2}, \Omega_{3}, \Omega_{4}$ reflects particular characteristics of the adaptive numerical approximation of (1.1): No significant local refinement is expected to occur in case of the convex domain $\Omega_{1}$ which has also been computed on uniform triangulations in [19]. This is different for the $L$-shaped domain $\Omega_{2}$. The reentrant corner at the origin presumably limits the regularity of the exact solution and therefore requires a higher resolution in its neighborhood in order to obtain optimal approximations. The regular hexagon defined by $\Omega_{3}$ can be regarded as an approximation of a circular domain. In analogy to known explicit solutions of (1.1) in disks, an almost circular interface separating the two phases is expected for $\Omega_{3}$. The existence and approximation theory discussed in the previous section does not immediately apply to the case of the non-Lipschitz domain $\Omega_{4}$. Nevertheless, practical experience from elliptic problems suggests that adaptive finite element methods still provide accurate approximations. These expectations are confirmed by our numerical experiments.

Figure 5.3, 5.7, 5.11, and 5.15 display the sequences of triangulations generated by (AFEM) for the domains $\Omega_{1}, \Omega_{2}, \Omega_{3}$, and $\Omega_{4}$. For the domains $\Omega_{1}$ and $\Omega_{3}$ we do not observe a significant local refinement, while for $\Omega_{2}$ and $\Omega_{4}$ there is a strong local refinement towards the origin at which the domains have reentrant corners.

The Figures 5.4, 5.8, 5.12, and 5.16 display the quantities $\eta_{E}, \eta_{A}$, and $\eta_{G}$ versus the number of degrees of freedoms for sequences of uniformly and adaptively generated triangulations. We remark that $\eta_{E}$ and $\eta_{A}$ provide upper bounds for the square of the stress error and observe from the plots that these quantities decay to zero at different rates in the examples. For the domains $\Omega_{1}$ and $\Omega_{3}$ they converge at the same rate to zero while for $\Omega_{2}$ and $\Omega_{4}$ the adaptive mesh-refinement strategy leads to improved, nearly linear convergence rates for the error bounds. The quantities $\eta_{G}$ do not allow for a straight-line in the logarithmic scaling of the plots. This is in agreement with the expectation that $\eta_{G}$ cannot be a lower bound for (any power of) the stress error. Figures 5.5, 5.9, 5.13, and 5.17 show the quantities $\delta_{\ell}$ for $\ell=0,1,2, \ldots$ and uniform and adaptive mesh-refinement with a logarithmic scaling on both axis for the domains $\Omega_{1}, \Omega_{2}, \Omega_{3}$, and $\Omega_{4}$. We deduce a similar behavior as for the error estimators.

The volume fractions $\Lambda\left(\left|D u_{\ell}\right|\right)$ are defined through the numerical approximation $u_{\ell} \in V_{\ell}$ for $\ell=11$ by the function

$$
\Lambda(t)=\left\{\begin{array}{cc}
0 & \text { for } 0 \leq t \leq t_{1} \\
\frac{t_{2}-t}{t_{2}-t_{1}} & \text { for } t_{1} \leq t \leq t_{2} \\
1 & \text { for } t_{2} \leq 1
\end{array}\right.
$$

and displayed in Figures 5.6, 5.10, 5.14, and 5.18 for the domains $\Omega_{1}, \Omega_{2}, \Omega_{3}$, and $\Omega_{4}$. Strong convergence of this quantity can only be expected if the approximations $u_{\ell}$ converge strongly. Sufficient but very severe conditions for this based on stabilisation are stated in [2]. In all of the examples we observe an arrangement of the two materials consisting of an interior region, a boundary layer, and a small transition layer between the two regions. In order to make the transition layer better observable we plotted for each of the four examples the microstructure region consisting of those points $\left\{x \in \Omega: 0<\Lambda\left(\left|D u_{11}(x)\right|\right)<1\right\}=:\left\{0<\Lambda\left(D u_{11}\right)<1\right\}$ in the domain $\Omega$ where the function $\Lambda\left(\left|D u_{11}\right|\right)$ is neither 0 nor 1 . 
The overall experience with (AFEM) for this degenerated minimisation problem from topology optimisation at hand is that local mesh-refinement is enforced as in strictly convex minimisation problems. A strong local refinement towards the interface separating the two phases does not arise in all of our numerical experiments and does not appear a necessity for linear convergence of the stress error. We conjecture that to be different for higher-order finite element methods.

\section{REFERENCES}

[1] Allaire, G. (2002). Shape optimization by the homogenization method. Springer-Verlag, New York.

[2] Bartels, S., Carstensen, C., Plecháč, P., Prohl, A. (2004). Convergence for stabilisation of degenerately convex minimisation problems. Interfaces Free Bound. 6, 2, 253-269.

[3] Binev, P., Dahmen, W., DeVore, R. (2004). Adaptive finite element methods with convergence rates. Numer. Math., 97, 2, 219-268.

[4] Brenner, S.C., Carstensen, C. (2004). Finite Element Methods. Chapter 4 in Encyclopedia of Computational Mechanics, John Wiley and Sons, 2004.

[5] Carstensen, C. (2004). Some remarks on the history and future of averaging techniques in a posteriori finite element error analysis. ZAMM Z. Angew. Math. Mech., 84, 1, 3-21.

[6] Carstensen, C. (2004). Convergence of adaptive finite element methods in computational mechanics. Proceedings of the Sixth World Congress on Computational Mechanics, September 2004.

[7] Carstensen, C. (2004). An adaptive mesh-refining algorithm allowing for an $H^{1}$-stable $L^{2}$-projection onto Courant finite element spaces. Constr. Approx. Vol. 20, No. 4, 549-564.

[8] Carstensen, C., Bartels, S. (2002). Each averaging technique yields reliable a posteriori error control in FEM on unstructured grids. I. Low order conforming, nonconforming, and mixed FEM. Math. Comp. 71, No. 239, 945-969.

[9] Carstensen, C., Jochimsen, K. (2003). Adaptive finite element methods for microstructures? Numerical experiments for a 2-well benchmark. Computing, 71, 2, 175-204.

[10] Carstensen, C., Müller, S. (2002). Local stress regularity in scalar nonconvex variational problems. SIAM J. Math. Anal., 34, 2, 495-509.

[11] Carstensen, C., Orlando, A., Valdman, J. (2006). A convergent adaptive finite element method for the primal problem of elastoplasticity. Internat. J. Numer. Methods Engrg. (in press). Early view version available through DOI 10.1002/nme.1686.

[12] Carstensen, C., Plecháč, P. (1997). Numerical solution of the scalar double-well problem allowing microstructure. Math. Comp., 66, 219, 997-1026.

[13] Carstensen, C., Plecháč, P. (2000). Numerical analysis of compatible phase transitions in elastic solids. SIAM J. Numer. Anal., 37, 6, 2061-2081.

[14] Cherkaev, A. (2000) Variational methods for structural optimization Springer-Verlag, New York.

[15] Dörfler, W. (1996). A convergent adaptive algorithm for Poisson's equation. SIAM J. Numer. Anal., 33, $3,1106-1124$.

[16] French, D.A. (1990). On the convergence of finite-element approximations of a relaxed variational problem. SIAM J. Numer. Anal. 27, 2, 419-436.

[17] Goodman, J., Kohn, R.V., Reyna, L. (1986). Numerical study of a relaxed variational problem from optimal design. Comput. Methods Appl. Mech. Engrg., 57, 1, 107-127.

[18] Hiriart-Urruty, J.-B., Lemaréchal, C. (2001). Fundamentals of convex analysis. Grundlehren Text Editions. Springer-Verlag, Berlin.

[19] Kawohl, B., Stara, J., Wittum, G. (1991). Analysis and numerical studies of a shape design problem. Archive for Rational Mechanics, 114, 349-363.

[20] Kohn, R., Strang, G. (1986). Optimal design and relaxation of variational problems I, II, III. Comm. Pure Appl. Math. 39 pp. 113-137, 139-182, 353-377.

[21] Morin, P., Nochetto, R.H., Siebert, K.G. (2000). Data oscillation and convergence of adaptive FEM. SIAM J. Numer. Anal., 38, 2, 466-488. 

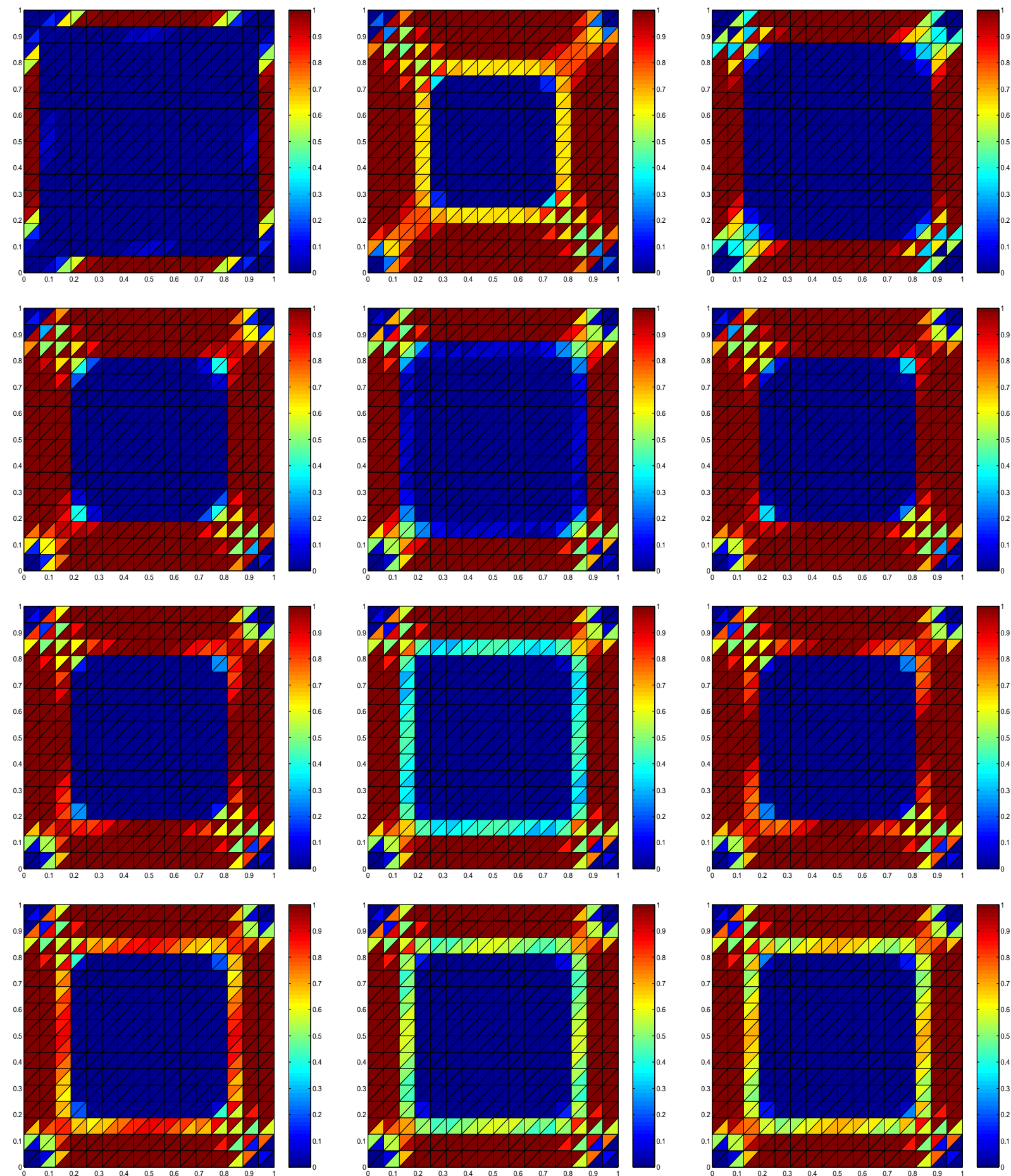

FiguRE 5.1. Sequence of volume fractions corresponding to solutions $u_{h}$ for new interval ends in the golden section search for the determination of the optimal $\lambda$ in case of domain $\Omega_{1}$. 

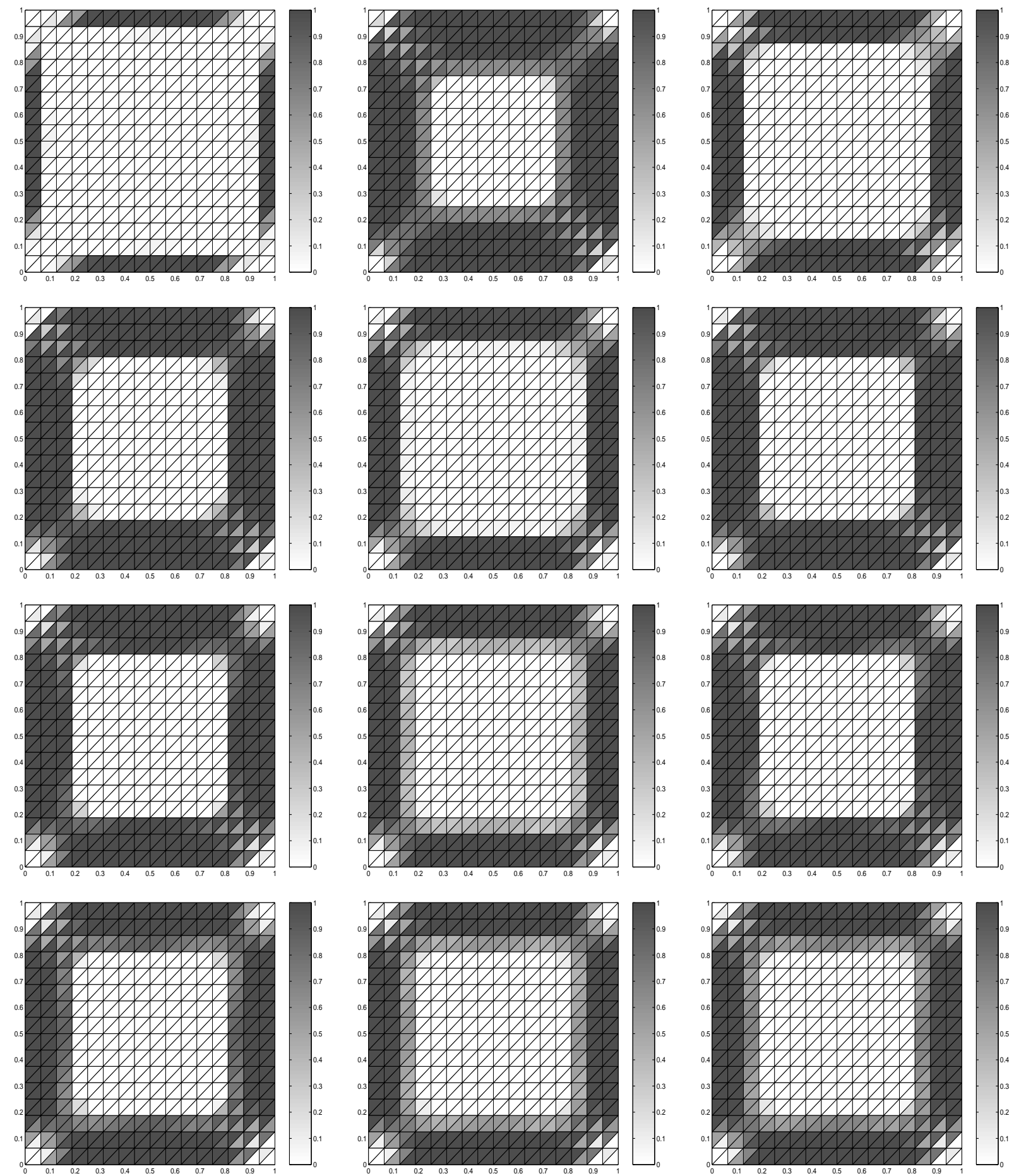

Figure 5.2. Sequence of volume fractions corresponding to solutions $u_{h}$ for new interval ends in the golden section search for the determination of the optimal $\lambda$ in case of domain $\Omega_{1}$ (in gray shading). 
[22] Morin, P., Nochetto, R.H., Siebert, K.G. (2003). Local problems on stars: a posteriori error estimators, convergence, and performance. Math. Comp., 72, 243, 1067-1097.

[23] Murat, F., Tartar, L. (1985). Optimality conditions and homogenization, in: Nonlinear Variational Problems. Eds.: A. Marino, L. Modica, S. Spagnolo and M. Degiovanni, Pitman Research Notes in Math. 127 pp. 1-8.

[24] Murat, F., Tartar, L. (1985). Calcul des variations et homogenization, in: Les méthodes de l'homogeneisation: théorie et applications en physique. Eds.: D. Bergman et al. Collection de la Direction des Études et Recherches d'Electricité de France 57 pp. 319-369.

[25] Veeser, A. (2002). Convergent adaptive finite elements for the nonlinear Laplacian. Numer. Math., 92, 4, 743-770.

[26] Zeidler, E. (1988). Nonlinear functional analysis and its applications. IV Applications to Mathematical Physics. Springer-Verlag, New York. 

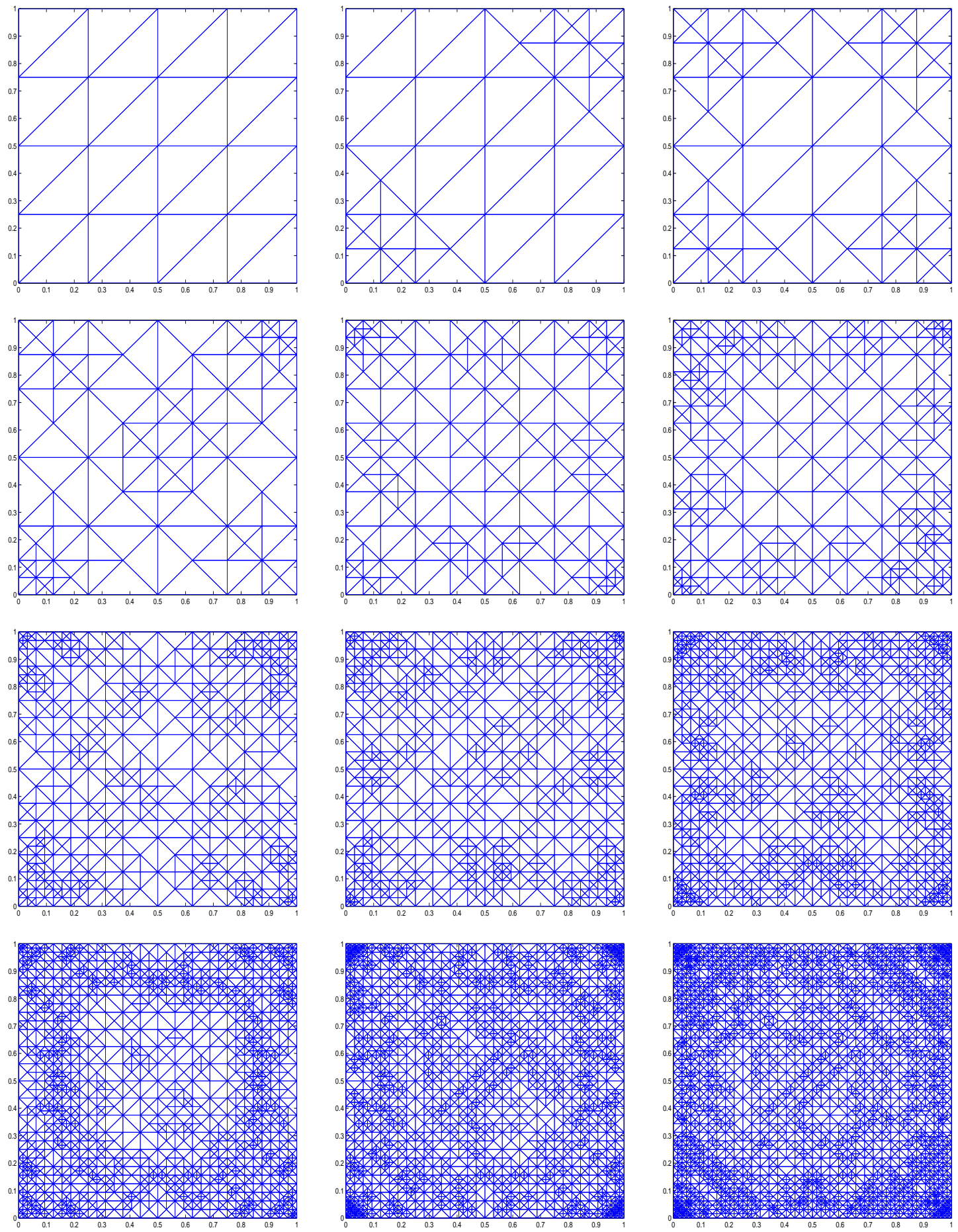

FiguRE 5.3. Adaptively generated triangulations $\mathcal{T}_{\ell}$ for $\ell=0,1,2, \ldots, 11$ for $\Omega_{1}$. 


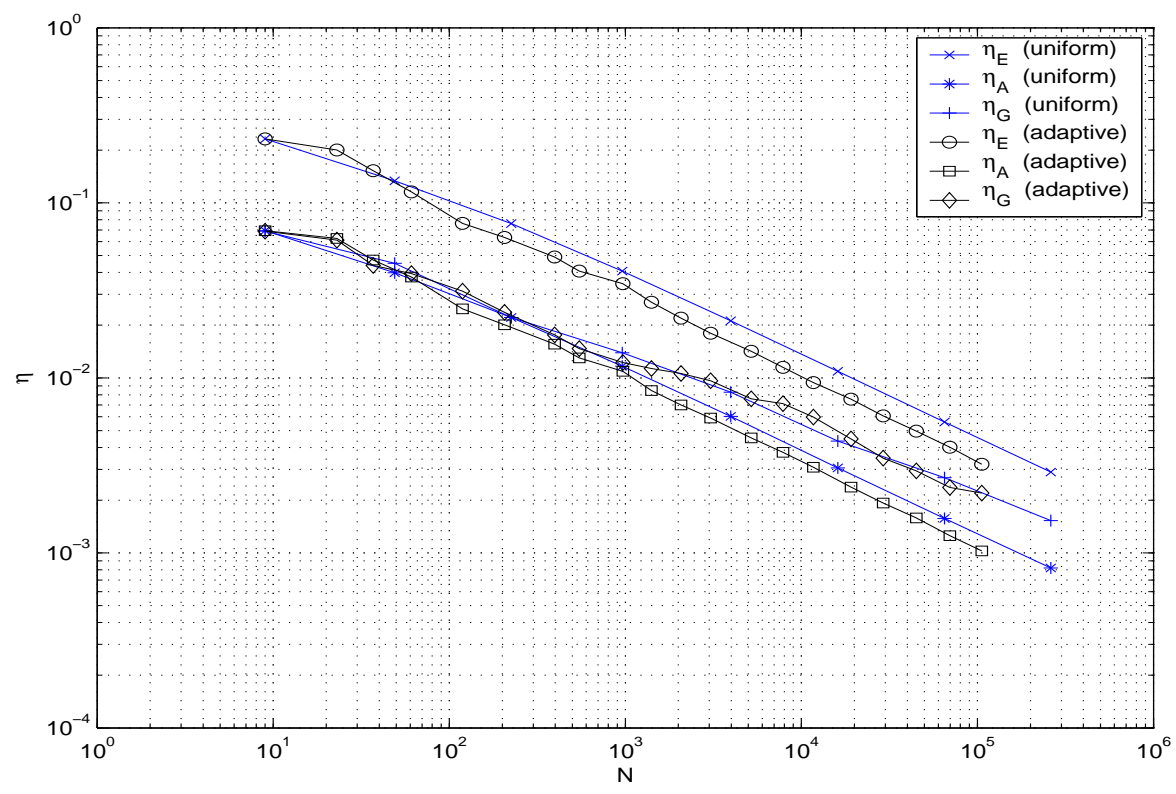

FigURE 5.4. Error estimators $\eta_{E}, \eta_{A}$, and $\eta_{G}$ versus number of degrees of freedom for $\Omega_{1}$.

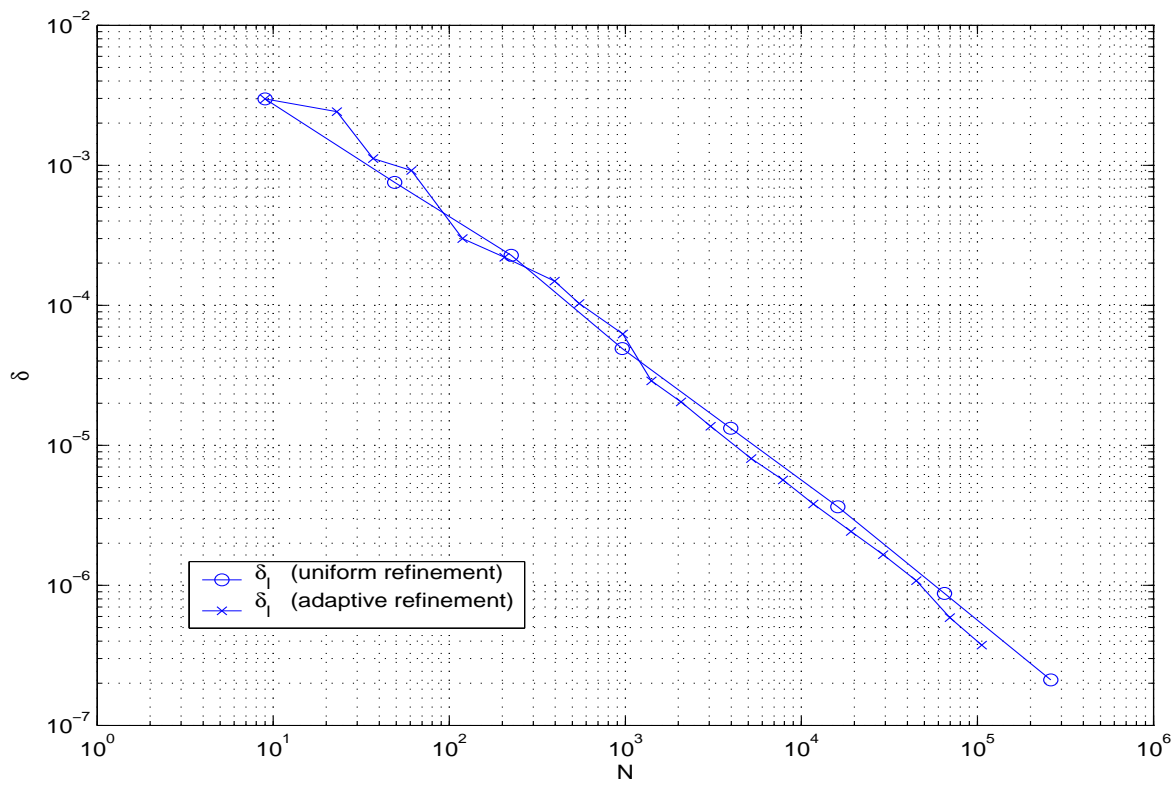

FiguRE 5.5. Decay of $\delta_{\ell}$ on the sequence of adaptively generated triangulations $\mathcal{T}_{\ell}$ for $\ell=0,1,2, \ldots, 19$ for $\Omega_{1}$. 

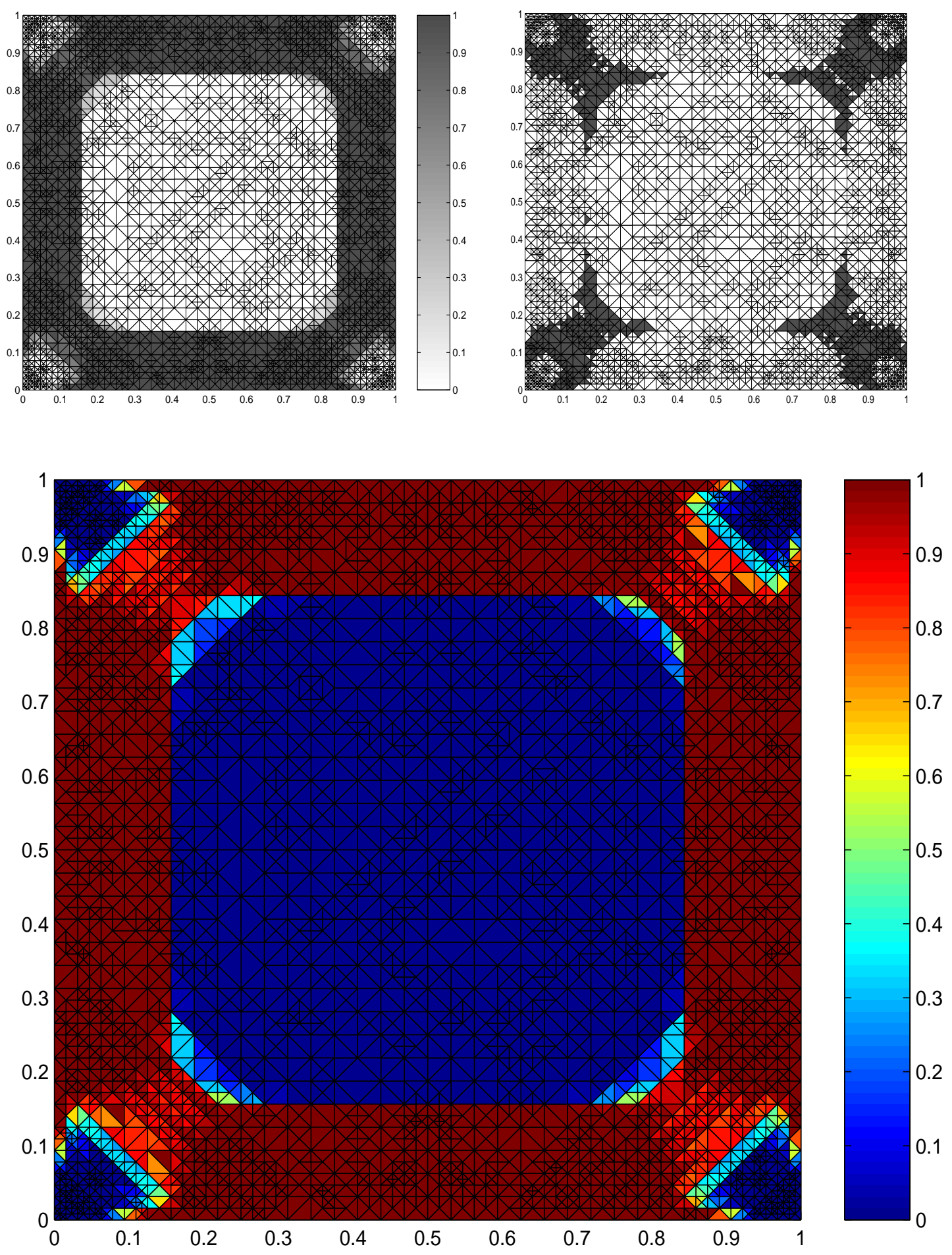

FiguRE 5.6. Volume fractions (upper left and lower plot) and microstructure region $\left\{0<\Lambda\left(\left|D u_{11}\right|\right)<1\right\}$ plotted in a darker shading (upper right plot) on $\mathcal{T}_{11}$ for $\Omega_{1}$. 

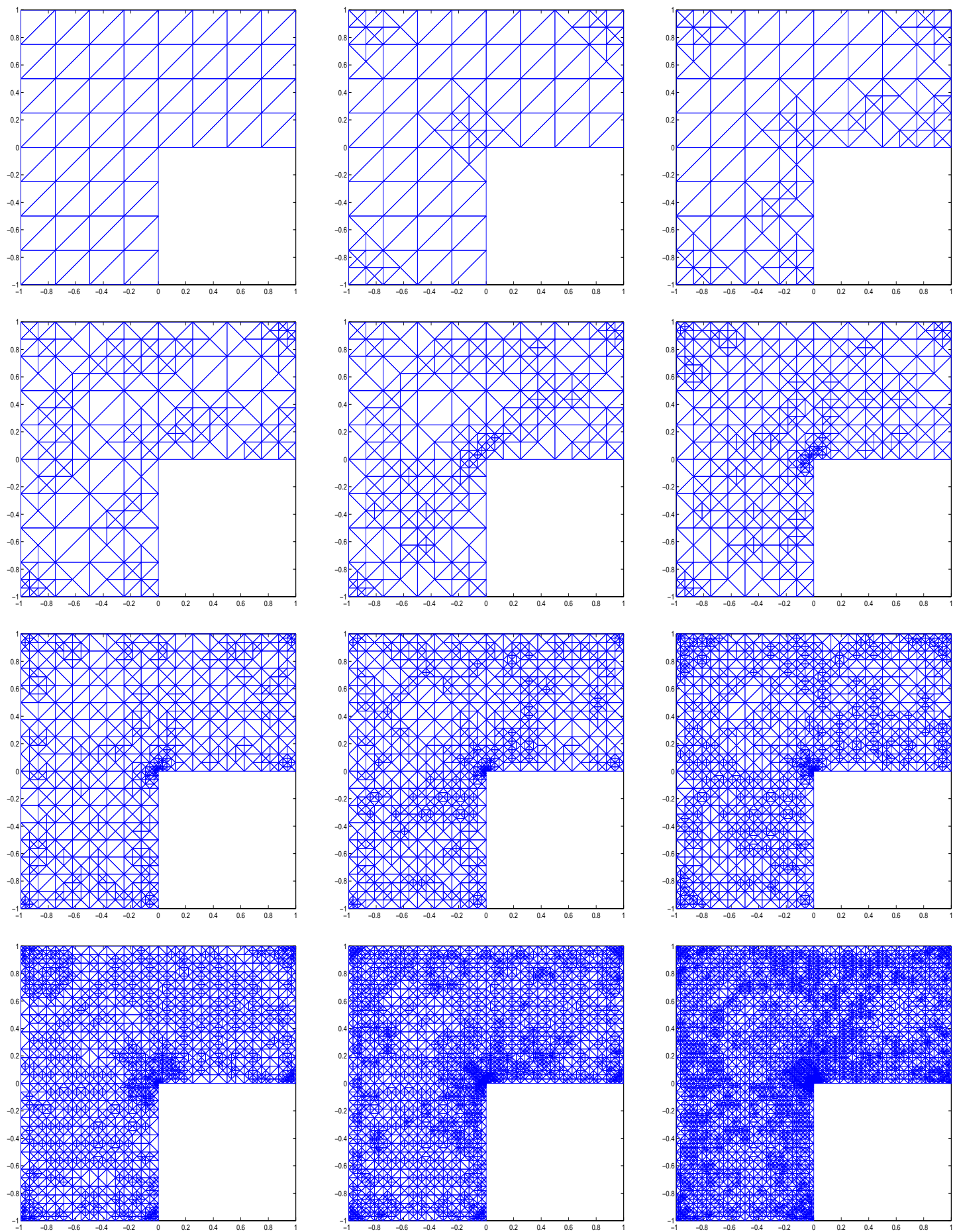

Figure 5.7. Adaptively generated triangulations $\mathcal{T}_{\ell}$ for $\ell=0,1,, 2, \ldots, 11$ for $\Omega_{2}$. 


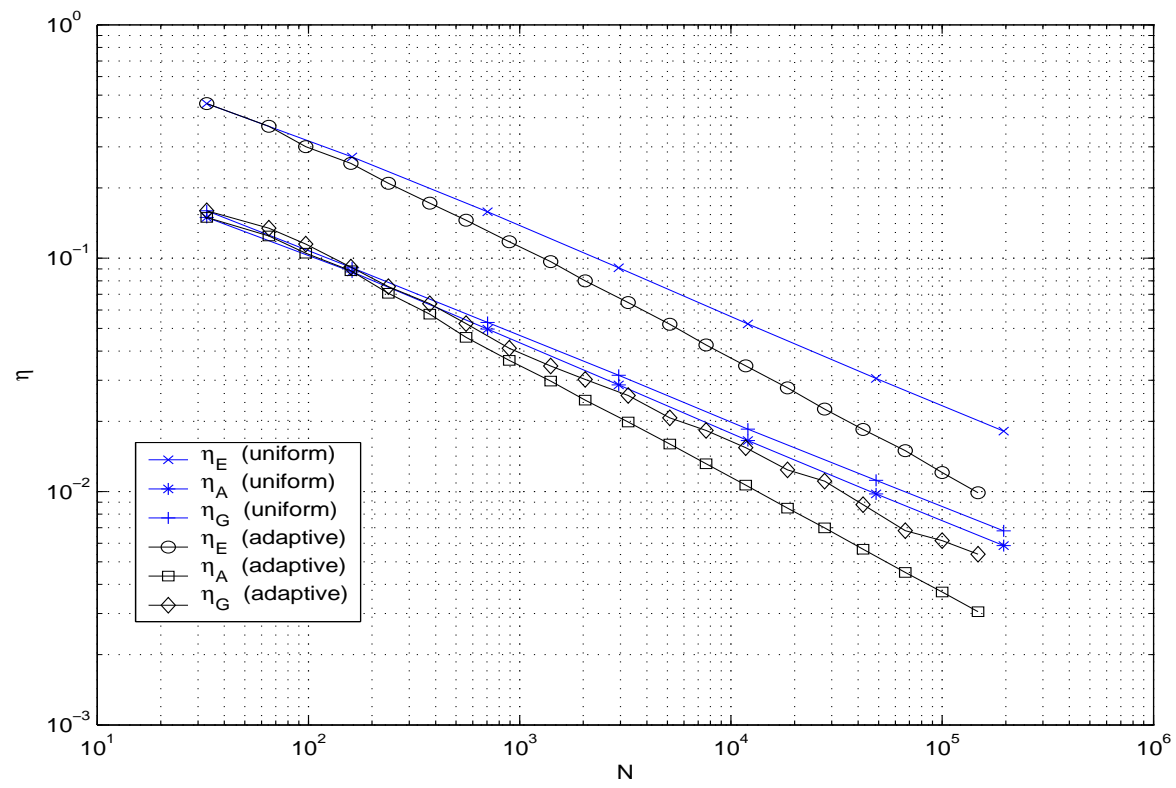

FiguRE 5.8. Error estimators $\eta_{E}, \eta_{A}$, and $\eta_{G}$ versus number of degrees of freedom for $\Omega_{2}$.

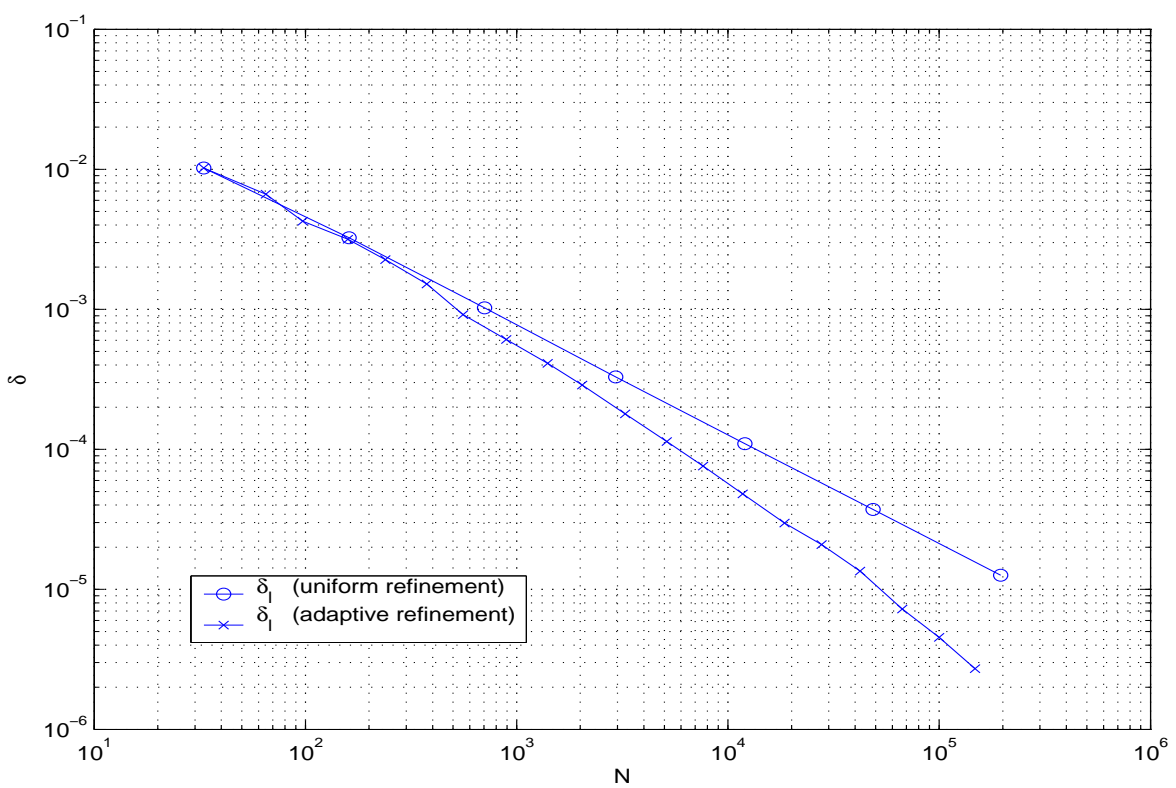

FigurE 5.9. Decay of $\delta_{\ell}$ on the sequence of adaptively generated triangulations $\mathcal{T}_{\ell}$ for $\ell=0,1,2, \ldots, 19$ for $\Omega_{2}$. 

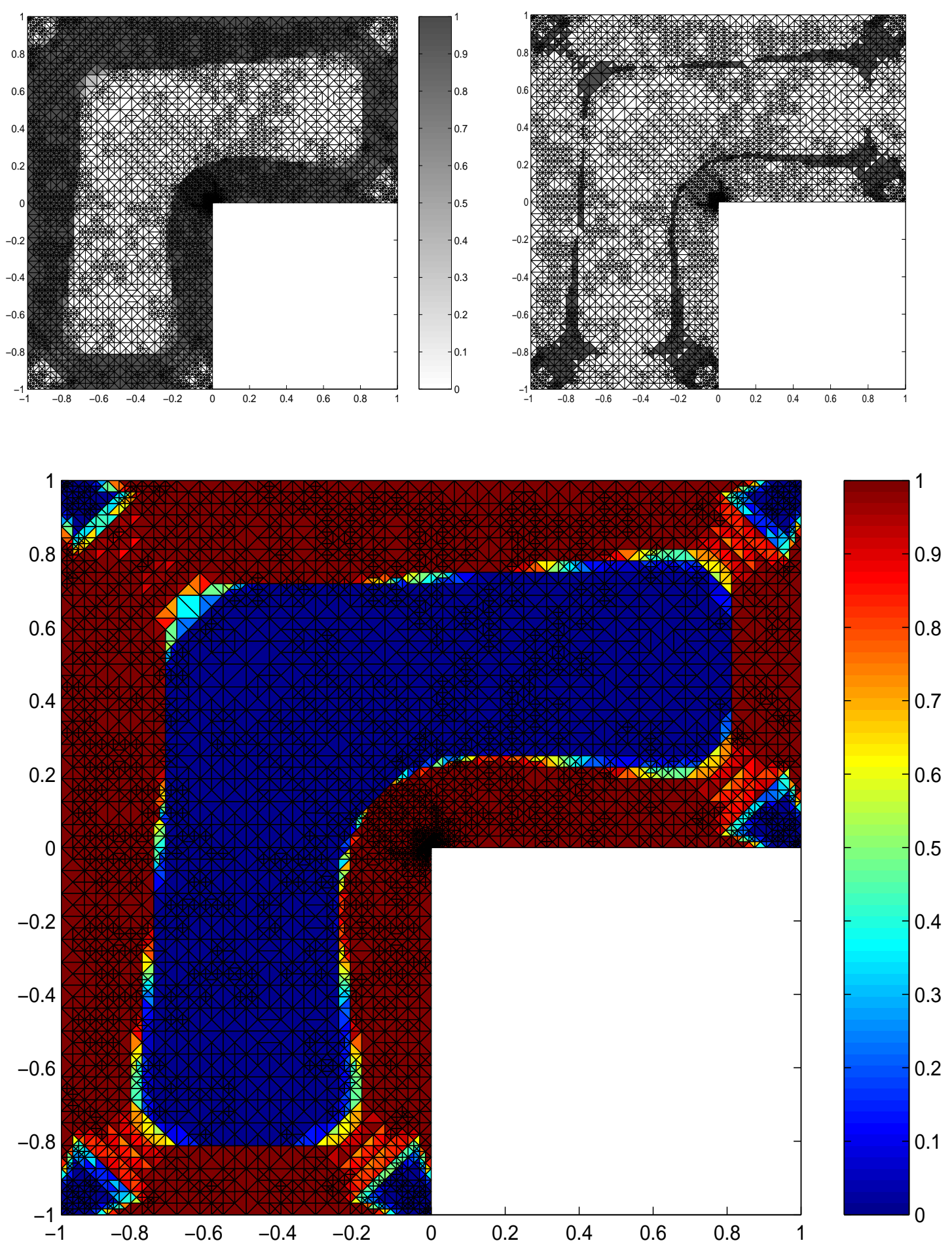

FiguRE 5.10. Volume fractions (upper left and lower plot) and microstructure region $\left\{0<\Lambda\left(\left|D u_{11}\right|\right)<1\right\}$ plotted in a darker shading (upper right plot) on $\mathcal{T}_{11}$ for $\Omega_{2}$. 

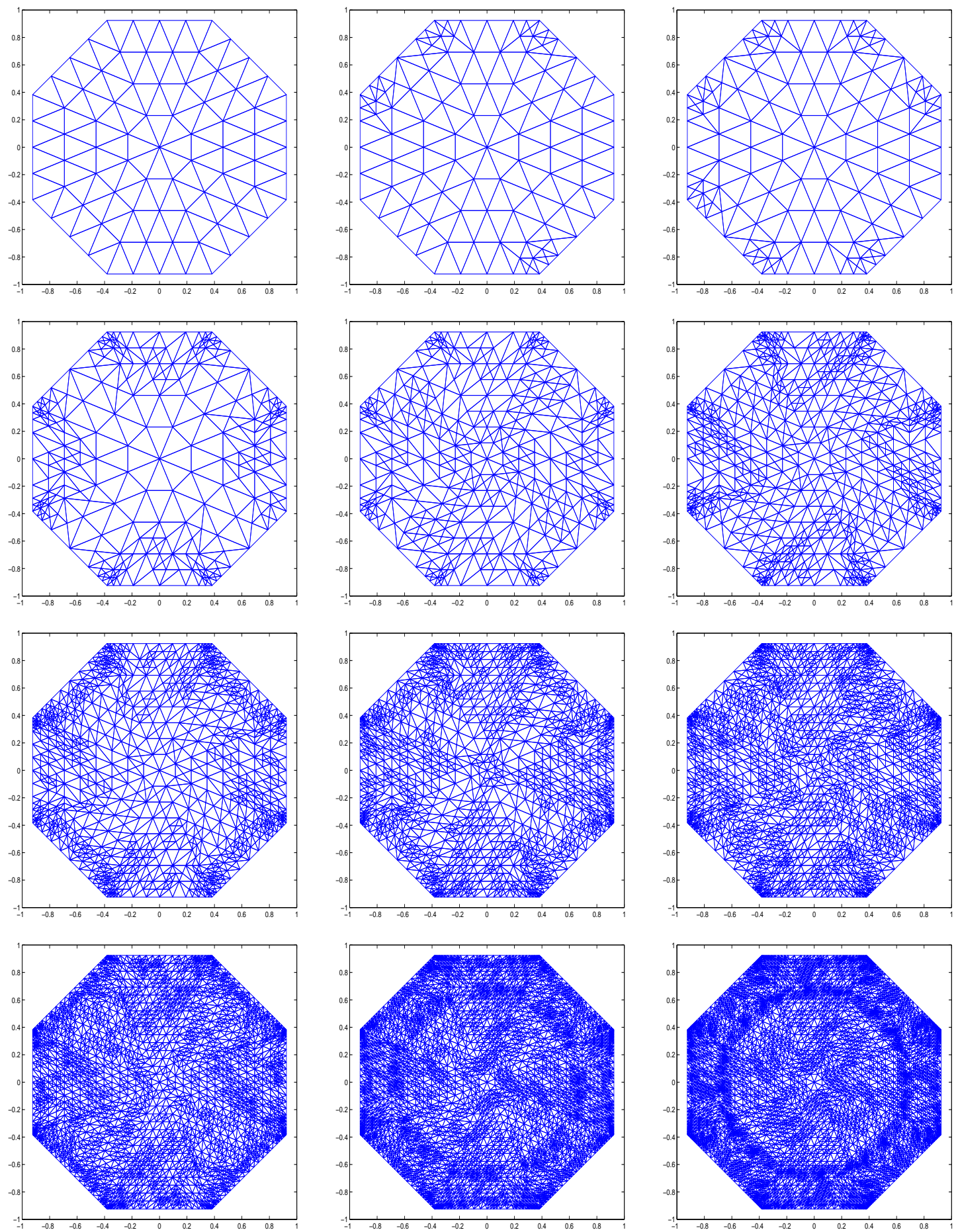

FiguRE 5.11. Adaptively generated triangulations $\mathcal{T}_{\ell}$ for $\ell=0,1,2, \ldots, 11$ for $\Omega_{3}$. 


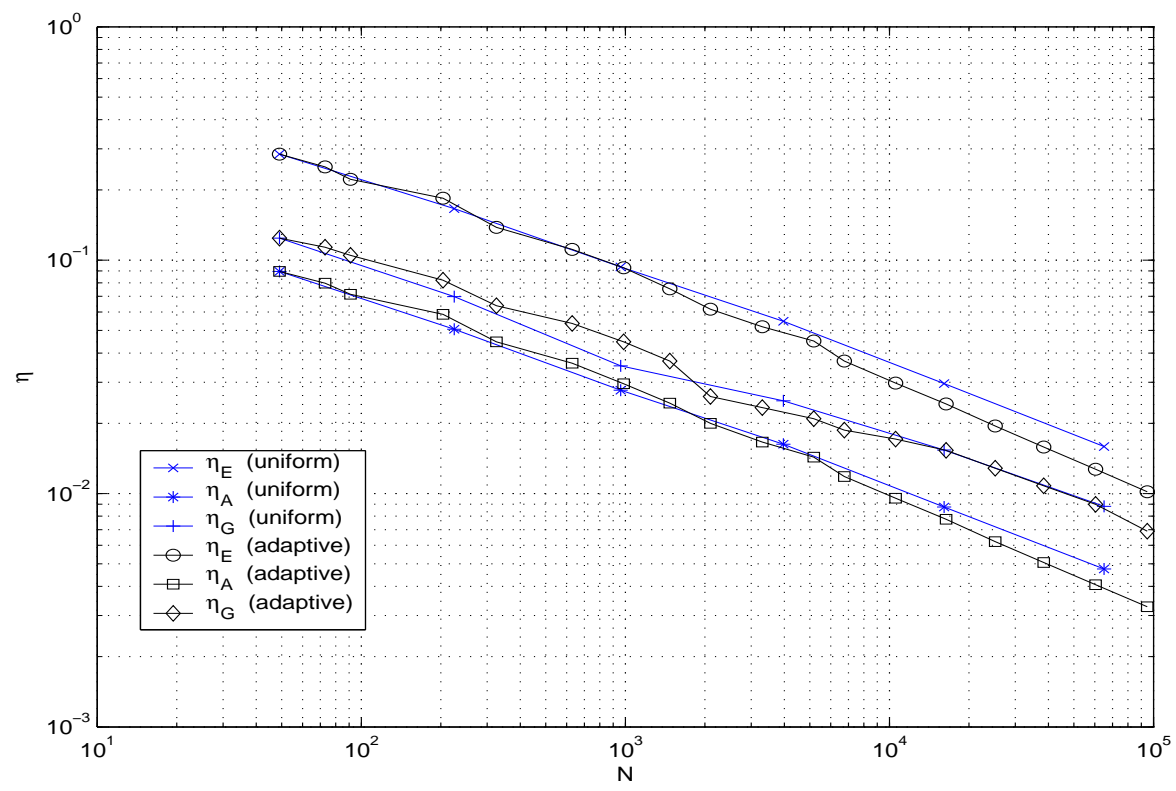

FigURE 5.12. Error estimators $\eta_{E}, \eta_{A}$, and $\eta_{G}$ versus number of degrees of freedom for $\Omega_{3}$.

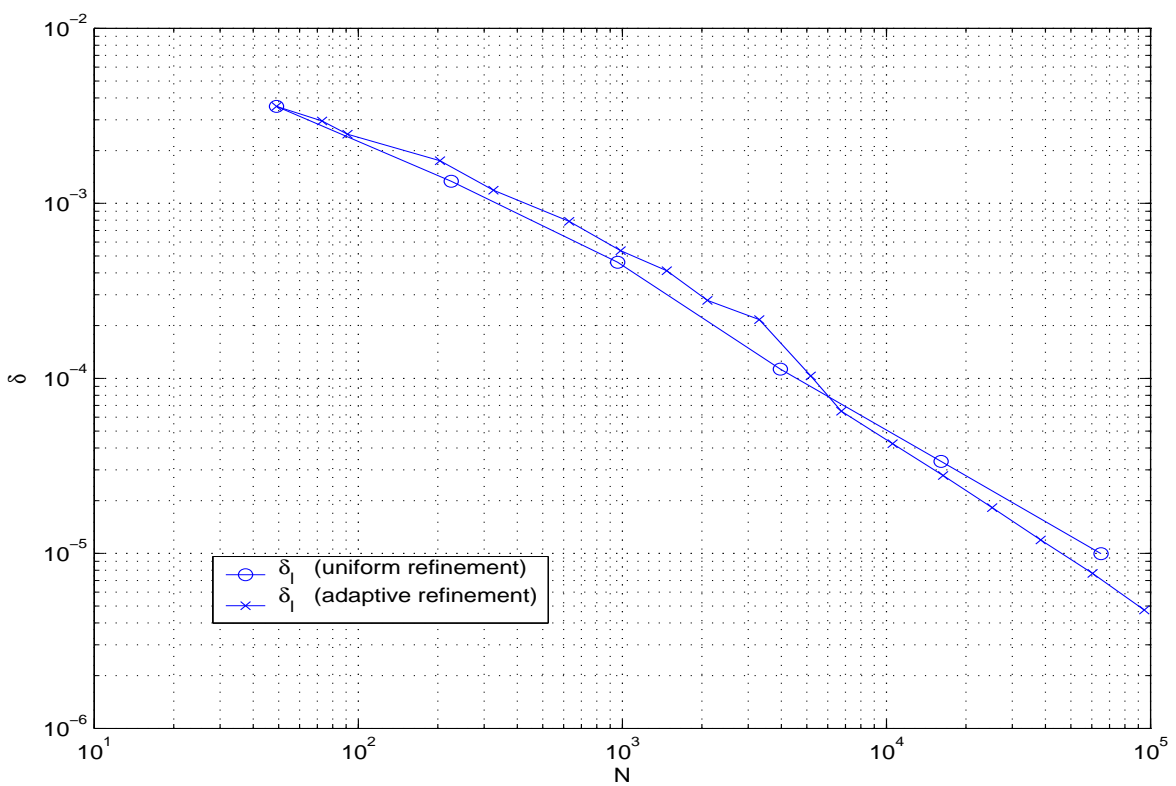

FiguRE 5.13. Decay of $\delta_{\ell}$ on the sequence of adaptively generated triangulations $\mathcal{T}_{\ell}$ for $\ell=0,1,2, \ldots, 17$ for $\Omega_{3}$. 

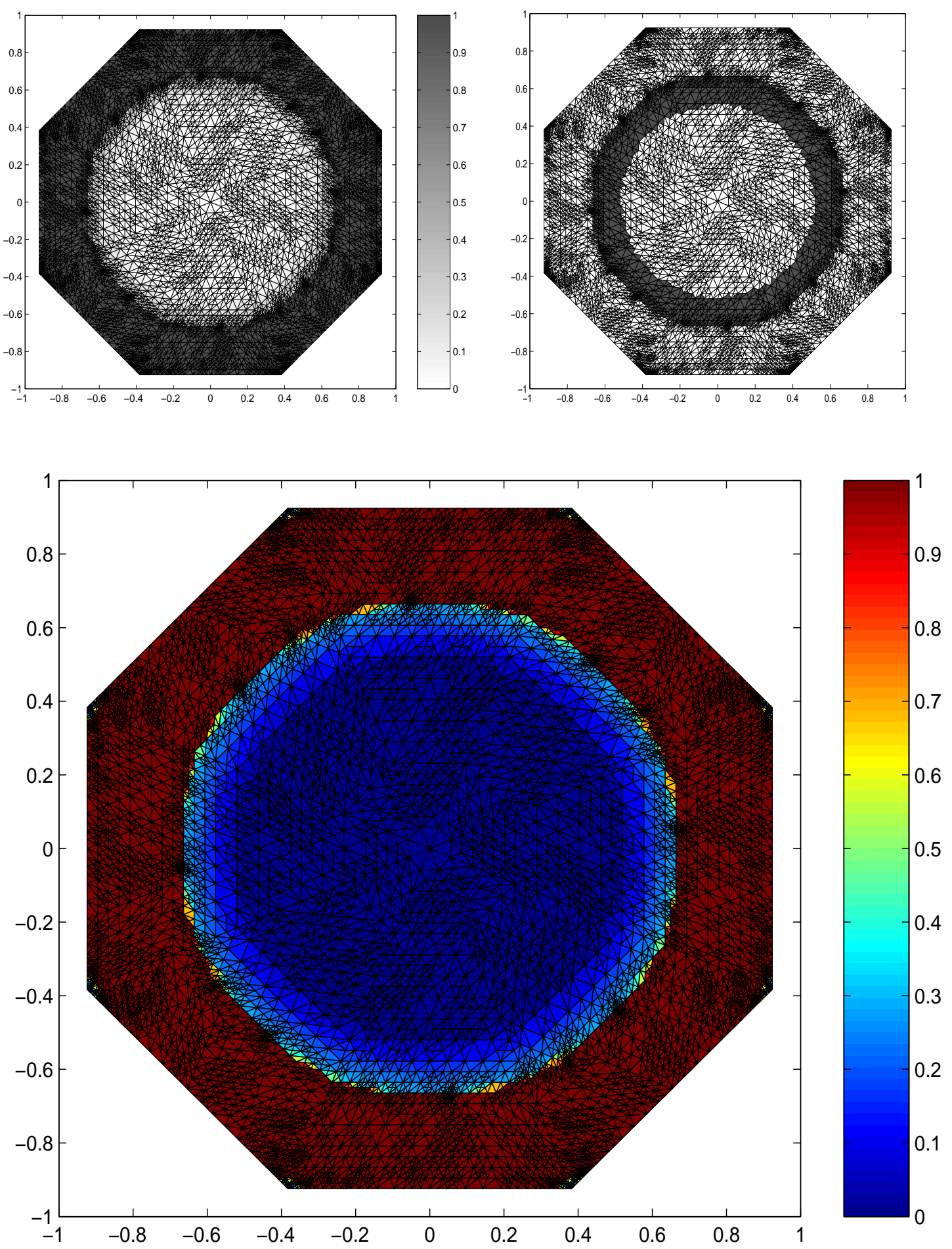

FigURE 5.14. Volume fractions (upper left and lower plot) and microstructure region $\left\{0<\Lambda\left(\left|D u_{11}\right|\right)<1\right\}$ plotted in a darker shading (upper right plot) on $\mathcal{T}_{11}$ for $\Omega_{3}$. 

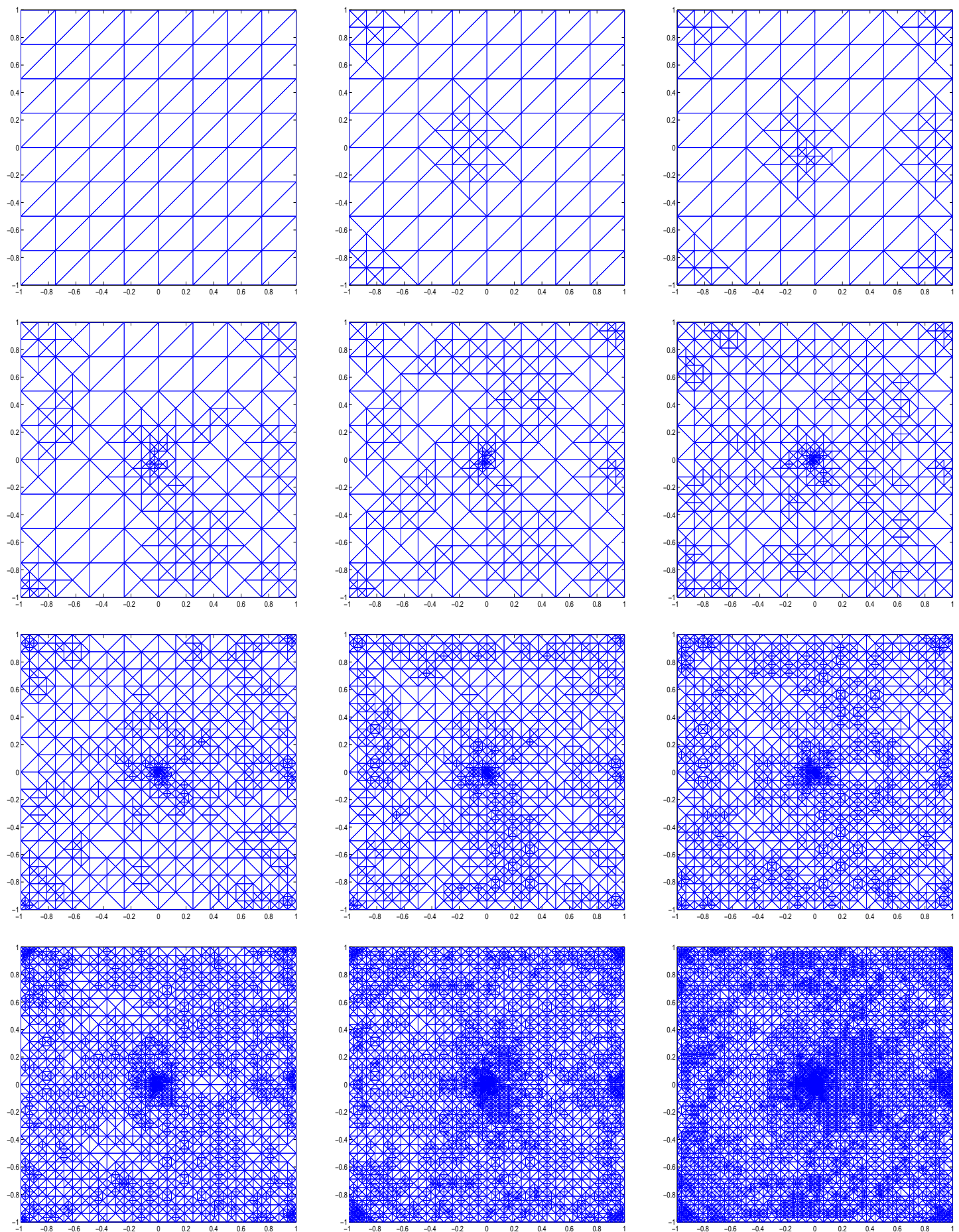

FiguRE 5.15. Adaptively generated triangulations $\mathcal{T}_{\ell}$ for $\ell=0,1,2, \ldots, 11$ for $\Omega_{4}$. 


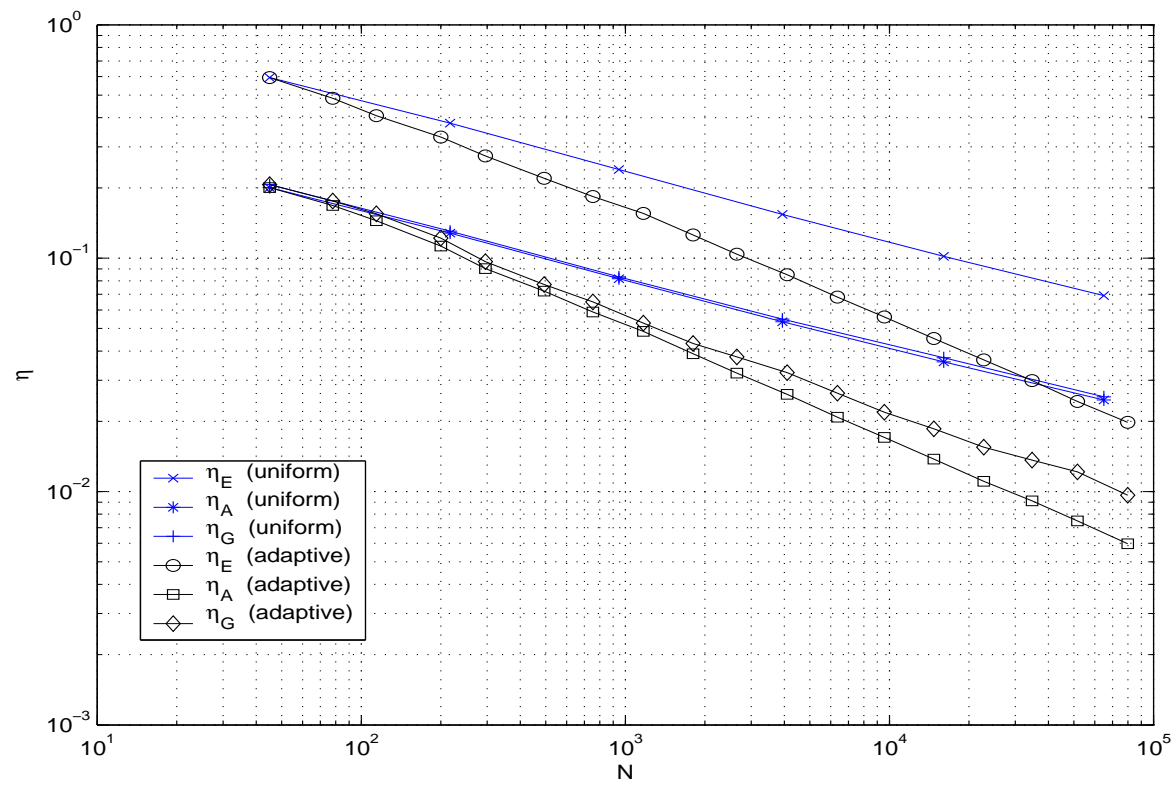

FigURE 5.16. Error estimators $\eta_{E}, \eta_{A}$, and $\eta_{G}$ versus number of degrees of freedomfor $\Omega_{4}$.

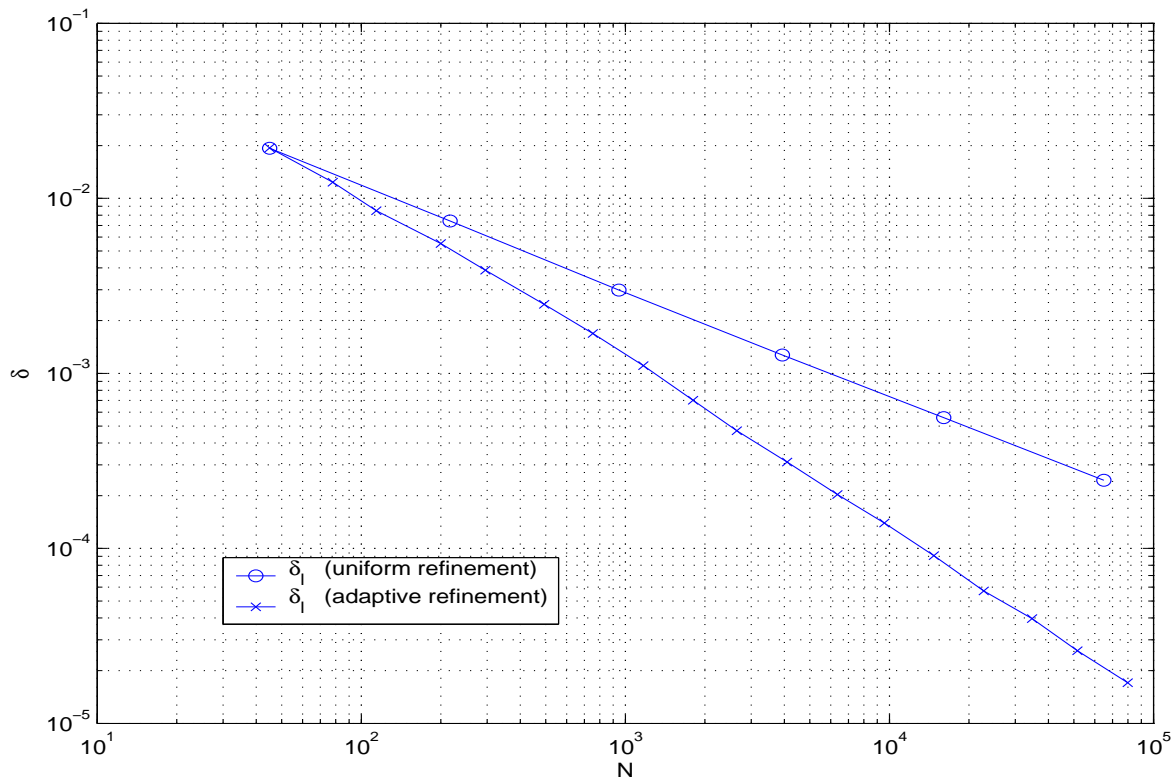

FiguRE 5.17. Decay of $\delta_{\ell}$ on the sequence of adaptively generated triangulations $\mathcal{T}_{\ell}$ for $\ell=0,1,2, \ldots, 17$ for $\Omega_{4}$. 

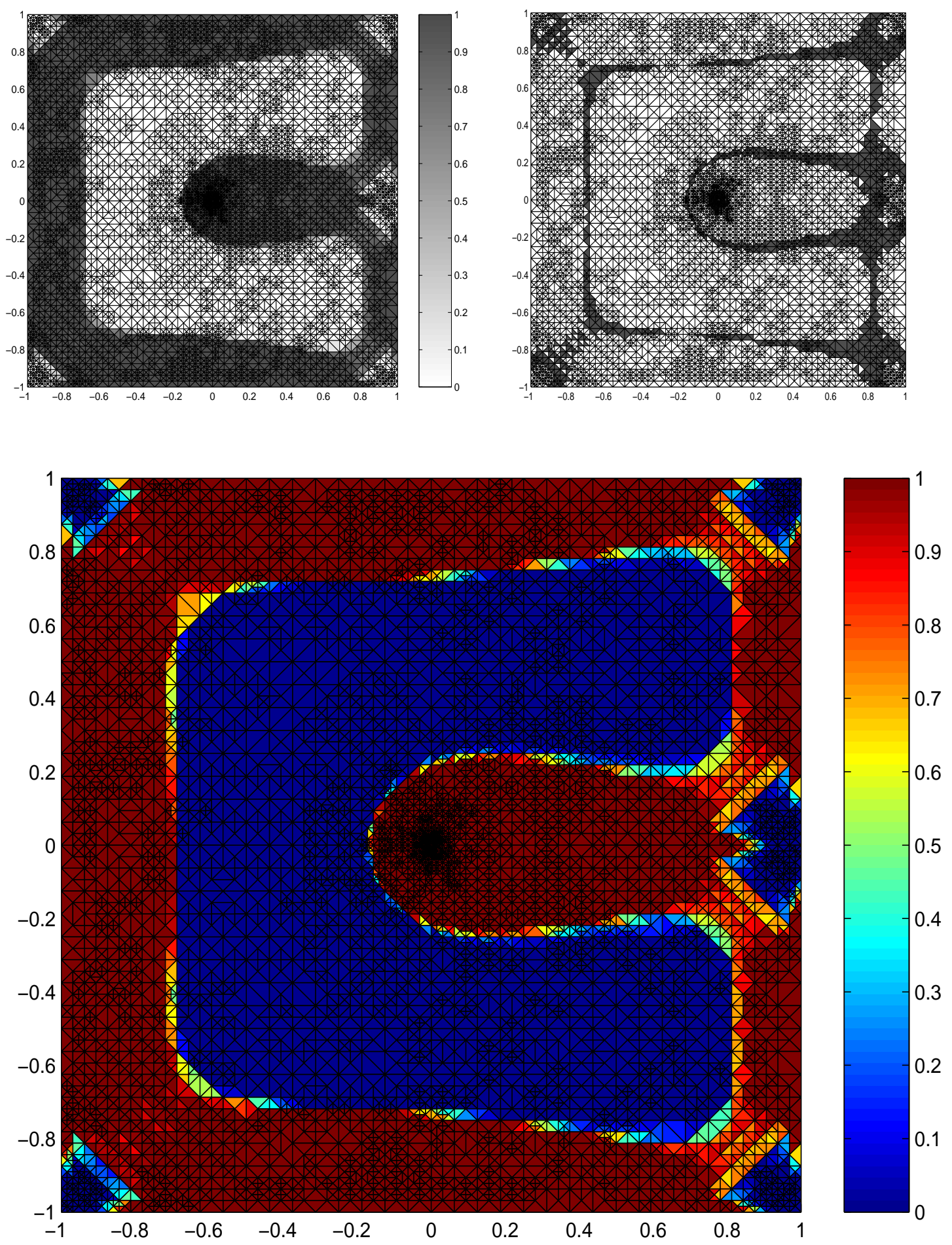

FiguRE 5.18. Volume fractions (upper left and lower plot) and microstructure region $\left\{0<\Lambda\left(\left|D u_{11}\right|\right)<1\right\}$ plotted in a darker shading (upper right plot) on $\mathcal{T}_{11}$ for $\Omega_{4}$. 